\title{
Endothelial ERK signaling controls lymphatic fate specification
}

\author{
Yong Deng, ${ }^{1}$ Deepak Atri, ${ }^{1}$ Anne Eichmann, ${ }^{1,2}$ and Michael Simons ${ }^{1,3}$ \\ ${ }^{1}$ Yale Cardiovascular Research Center, Section of Cardiovascular Medicine, Department of Internal Medicine, Yale University School of Medicine, \\ New Haven, Connecticut, USA. ${ }^{2}$ Center for Interdisciplinary Research in Biology (CIRB), Collège de France, \\ Paris, France. ${ }^{3}$ Department of Cell Biology, Yale University School of Medicine, New Haven, Connecticut, USA.
}

\begin{abstract}
Lymphatic vessels are thought to arise from PROX1-positive endothelial cells (ECs) in the cardinal vein in response to induction of SOX18 expression; however, the molecular event responsible for increased SOX18 expression has not been established. We generated mice with endothelial-specific, inducible expression of an $R A F 1$ gene with a gain-of-function mutation $\left(R A F 1^{S 259 A}\right)$ that is associated with Noonan syndrome. Expression of mutant RAF1 ${ }^{\text {S259A }}$ in ECs activated ERK and induced SOX18 and PROX1 expression, leading to increased commitment of venous ECs to the lymphatic fate. Excessive production of lymphatic ECs resulted in lymphangiectasia that was highly reminiscent of abnormal lymphatics seen in Noonan syndrome and similar "RASopathies." Inhibition of ERK signaling during development abrogated the lymphatic differentiation program and rescued the lymphatic phenotypes induced by expression of $R A F 1^{5259 A}$. These data suggest that ERK activation plays a key role in lymphatic EC fate specification and that excessive ERK activation is the basis of lymphatic abnormalities seen in Noonan syndrome and related diseases.
\end{abstract}

\section{Introduction}

The lymphatic system is composed of a network of blind-ended, thin-walled capillaries and larger vessels that drain protein-rich interstitial fluid from the extracellular spaces. It plays a critical role in fluid regulation, immune response, and tumor metastasis. Defects in the lymphatic system, congenital as well as acquired, are encountered in a number of disease states (1-4). One of the more common and least understood lymphatic defects is lymphangiectasia, a pathological dilation of dysmorphic lymphatic vasculature that can lead to lymphedema and compression of nearby structures $(5,6)$. Lymphatic defects such as lymphangiectasia can be particularly prominent in patients with Noonan and LEOPARD syndromes, conditions characterized by gain-of-function mutations in the RAS/RAF signaling cascade $(7,8)$. The molecular basis of the lymphatic defects in these diseases is still unknown. Previously, we have demonstrated that under normal conditions, the PI3K/AKT signaling pathway inhibits ERK signaling via AKT1dependent phosphorylation of RAF1 on Ser259 (Figure 1, A and B) in endothelial cells (ECs) (9). Gain-of-function mutations of RAF1 at Ser259 have been frequently identified in Noonan syndrome patients (10-12). Here we set out to examine the specific effect of endothelial RAF1/ERK activation in the developing vasculature.

Mammalianlymphatic vessels originate from embryonicveins $(13,14)$. During early embryonic development, a subset of PROX1-positive ECs forms at E1 0.5 in the lateral portion of the cardinal veins. These cells then sprout laterally, starting at E11.5, to form lymph sacs $(1,13)$. Prox1 knockout embryos lack lymph sacs and lymphatic vessels (15), and Prox1-deficient ECs fail to express lymphatic endothelial markers and instead retain their blood vascular endothelial phenotype $(13,15)$. At later stages of development, PROX1 expression is reduced in veins and becomes restricted to the lymphatic vasculature (16).

The homeobox transcription factor SOX18 is transiently expressed in cardinal vein ECs prior to PROX1, and is required for initiation

Conflict of interest: The authors have declared that no conflict of interest exists. Citation for this article: J Clin Invest. 2013;123(3):1202-1215. doi:10.1172/JCI63034 of the lymphatic EC (LEC) differentiation program upstream of PROX1 (17). During LEC fate induction, SOX18 expression is not restricted to venous ECs, as it is also expressed in arterial ECs, which do not continue to express PROX1 $(16,17)$. In contrast to PROX1, SOX18 expression in the lymphatic vasculature is not detected during later stages of embryonic lymphangiogenesis, suggesting that SOX18 does not play a role in the maintenance of LEC identity (17). Two closely related group F Sox factors, SOX7 and SOX17, are able to functionally substitute for SOX18 in vitro and in vivo in a straindependent manner (18). However, neither of these factors is normally expressed during lymphatic development. Sox18-null embryos show a complete lack of PROX1-positive cells and LEC differentiation from the cardinal vein (17). Despite the critical role that SOX18 plays in developmental lymphangiogenesis, nothing is known about the molecular mechanisms controlling its expression.

Cardinal vein ECs that give rise to lymphatics express both VEGFR2 and VEGFR3, with the latter's ligand-binding and kinase activity considered to be required for lymphangiogenesis (19). Binding of VEGF-C to VEGFR3 leads to activation of PI3K/AKT and RAF1/MEK/ERK signaling pathways, with the former playing a critical role in regulation of LEC migration $(20,21)$. The role of ERK is suggested in studies demonstrating excessive LEC differentiation, subcutaneous hemorrhage, edema, and the presence of dilated lymphatic vessels in mice with deletions of the negative regulators of ERK signaling Spred1, Spred2, and Rasa1 (22, 23). Nevertheless, the role of ERK signaling in lymphatic development and its mechanism of action have not been established. Here, we used an endothelial-specific non-AKT suppressible RAF1 mutant transgenic mouse model to show that the RAF1/MEK/ERK signaling input regulates SOX18-induced LEC fate specification and developmental lymphangiogenesis.

\section{Results}

Generation of endothelial RAF1 gain-of-function mice. To fully explore the important role played by ERK signaling in the endothelium, we took advantage of the observation that $R A F 1^{S 259 \mathrm{~A}}$ 
A Extracellular signals

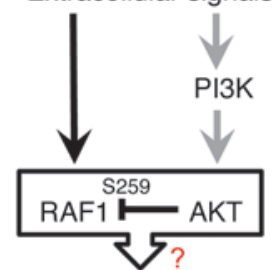

B

Vascular development

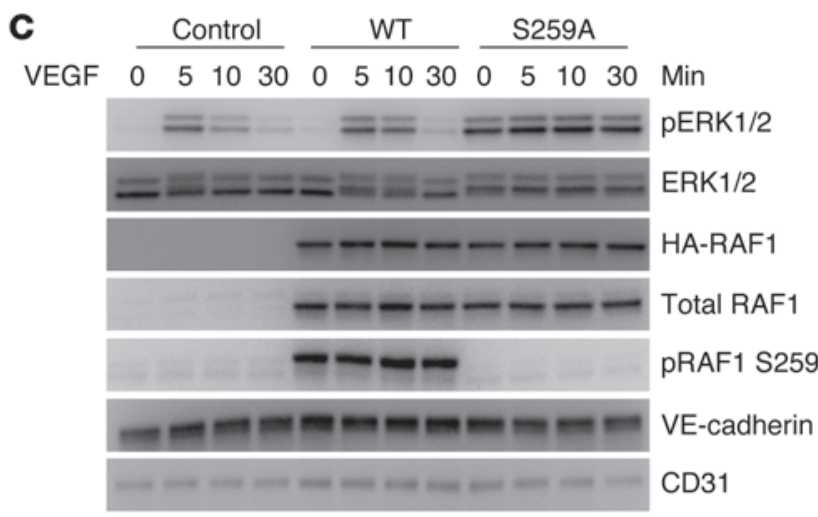

D
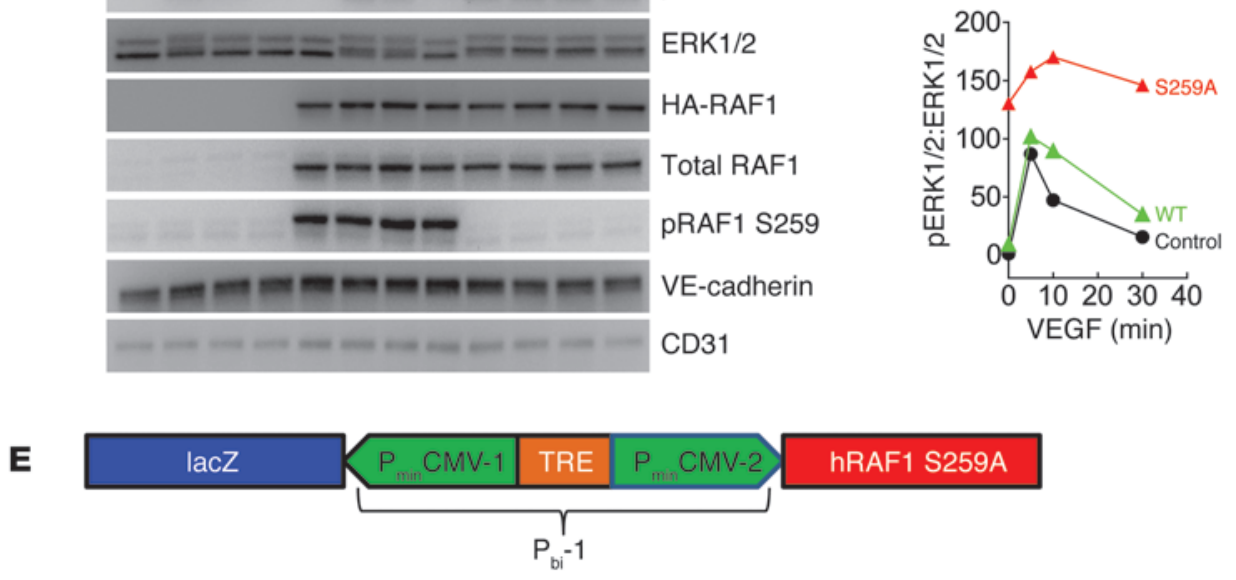

$\mathbf{F}$
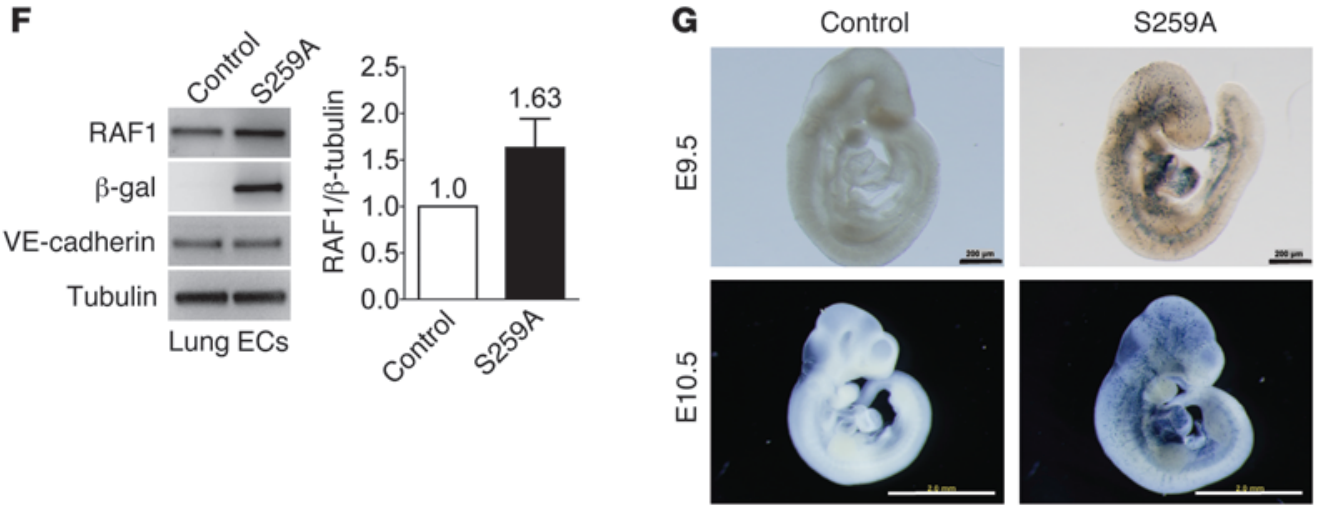

Figure 1

Endothelial-specific expression of RAF1 2259 A blocks RAF1-AKT crosstalk and activates ERK. (A) RAF1-AKT crosstalk. Upon extracellular signal stimulation, AKT phosphorylates RAF1 at Ser259 and inhibits RAF1 activation. (B) Scheme of RAF1 phosphorylation sites. (C) Western blot demonstrates ERK1/2 activation by RAF1S259A. Serum-starved HUVECs transduced with empty control, wild-type HA-RAF1 (WT), or HA-RAF1 ${ }^{S 259 A}$ (S259A) lentiviruses were stimulated with $50 \mathrm{ng} / \mathrm{ml} \mathrm{VEGF-A}_{164}$ for the indicated times. (D) ERK activation shown in (C) was quantified by densitometry and is represented as a ratio of $\mathrm{pERK} 1 / 2$ to total ERK1/2. (E) Scheme of construct for TRE-RAF1S259A transgenic mice. (F) Western blot showing RAF1 expression in purified primary lung ECs. Densitometry of RAF1 levels compared with those of $\beta$-tubulin is shown on the right. Data represent the mean \pm SEM of 3 independent experiments. (G) X-gal staining of E9.5 and E10.5 embryos. Scale bars: $200 \mu \mathrm{m}(\mathrm{E} 9.5)$ and $2 \mathrm{~mm}(\mathrm{E} 10.5)$.

expression leads to ERK activation (11). Consistent with these results, expression of a lentiviral $R A F 1^{\text {S259A }}$ construct in ECs also resulted in ERK activation (Figure 1, C and D). To explore the effect of ERK activation in the vasculature in vivo, endothelialspecific, inducible $R A F 1^{S 259 A}$ transgenic mice were generated by crossing a line with a bidirectional CMV promoter under the control of a tetracycline-responsive promoter element driving human $R A F 1^{S 259 A}$ and $L a c Z$ (TRE-RAF1 ${ }^{S 259 A}$ ) (Figure 1E) with
$V E$-cadherin-tTA mice (24). To confirm expression and determine the expression level of the transgene, we isolated lung ECs from $V E$-cadherin-tTA/RAF1 ${ }^{5259 A}$ double-transgenic (S259A) mice. Western blot analysis of RAF1 expression demonstrated a $63 \%$ increase in $R A F 1^{S 259 A}$ compared with wild-type ECs (Figure $1 \mathrm{~F}$ ). The endothelial-specific expression of the transgene was confirmed by whole-mount X-gal staining of E9.5 and E10.5 embryos (Figure 1G). 
A

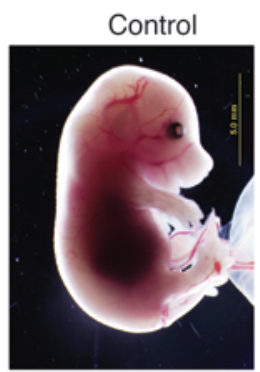

C

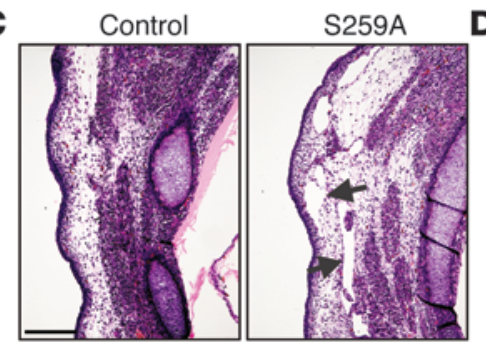

S259A

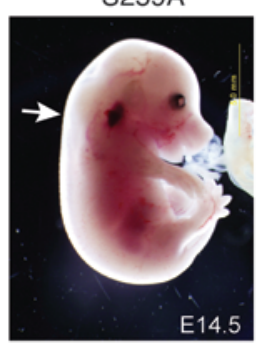

D

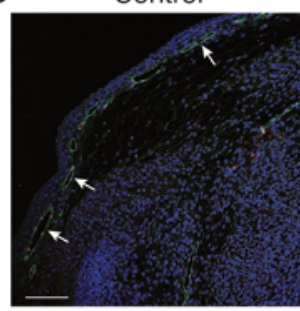

B

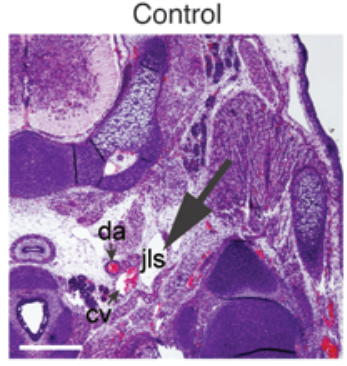

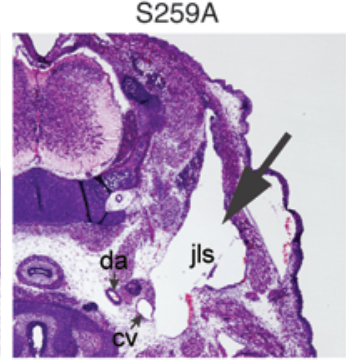

E
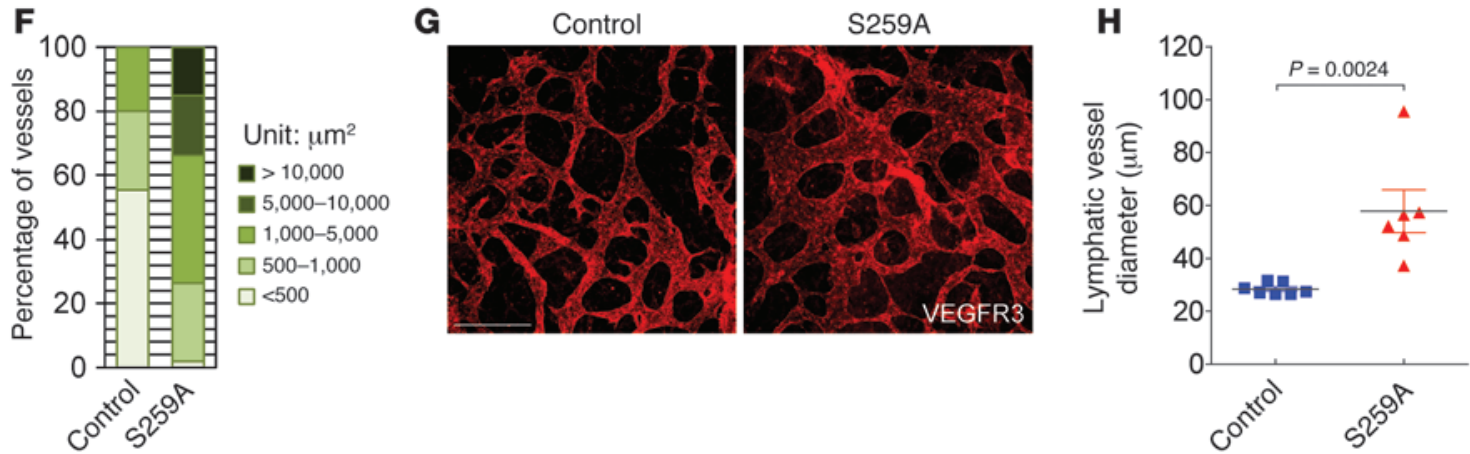

Figure 2

Endothelial-specific expression of RAF1S259A induces enlarged lymphatic vessels. (A) S259A embryos show edema (arrowhead) at E14.5. Scale bars: $5 \mathrm{~mm}$. (B) H\&E staining of E14.5 embryo sections revealed extremely enlarged jugular lymph sacs (arrows) in S259A embryos. Scale bar: 100 um. (C) H\&E staining of E14.5 embryo sections revealed enlarged subcutaneous vessels (arrows). Scale bar: $150 \mu \mathrm{m}$. (D) Immunofluorescence staining of E14.5 embryo sections revealed enlarged subcutaneous lymphatic vessels (arrows). VEGFR3 (green); PROX1 (red); DAPI (blue). Scale bar: $200 \mu \mathrm{m}$. (E) Quantitative analysis of subcutaneous lymphatic vessel lumen area of E14.5 embryos based on VEGFR3/PROX1 double staining shown in (D). Lumen areas of subcutaneous lymphatic vessels. Data represent the mean \pm SEM. (F) Distribution of subcutaneous lymphatic vessel lumen size. Subcutaneous lymphatic vessels shown in (D) were grouped based on different lumen sizes as indicated. Percentages of the number for each group out of the total number of vessels are shown. Data represent the mean of 4 embryos for each genotype. (G) VEGFR3 (red) whole-mount staining of E14.5 embryo dorsal skins. Scale bar: $200 \mu \mathrm{m}$. (H) Quantitative analysis of lymphatic vessel diameter based on VEGFR3 staining shown in (G). Control, $n=7$ embryos; S259A, $n=6$ embryos. Mean \pm SEM. cv, cardinal vein; da, descending aorta; jls, jugular lymph sac.

Of the 58 pups from the TRE-RAF1 $1^{S 259 A}$ and VE-cadherin-tTA cross, only 2 VE-cadherin-tTA/RAF1 ${ }^{\text {S259A }}$ double-transgenic (S259A) mice were born alive. X-gal staining showed trace expression (not shown) of the transgene, suggesting that endothelial expression of $R A F 1^{S 259 A}$ causes embryonic lethality. Analysis of developing embryos generated by timed mating showed that at E9.5, only a small portion of the ECs showed positive X-gal staining, while by E12.5, a majority of the ECs were X-gal-positive (data not shown). This suggests that the VE-cadherin promoter in this TET-OFF construct is not fully turned on until approximately E12.5, which is consistent with previous observations (24). Prior to E12.5, no significant defects were observed in the cardiovascular system of S259A embryos. However, at E14.5 these embryos showed a gross subcutaneous edema (Figure 2A), with nearly 100\% (53 of 55 embryos) lethality by E15.5. No hemorrhage was observed except for subcutaneous bleeding in the neck dorsally to the right ear in $50 \%$ of the embryos. Further histological analysis of E14.5 embryos showed a high prevalence of cardiac defects in S259A embryos, including ventricular hypertrabeculation and wall thinning (Supplemental Figure 1; supplemental material available online with this article; doi:10.1172/JCI63034DS1), which are associated with embryonic lethality (25). These findings are consistent with a high prevalence of cardiac defects in various "RASopathies" including Noonan syndrome $(11,26)$.

S259A mice develop lymphangiectasia. The extensive edema in S259A embryos suggests defective lymphatic development. H\&E staining of sections of E14.5 embryos revealed massively enlarged and malformed jugular lymphatic sacs (Figure 2B) and subcutaneous lym- 

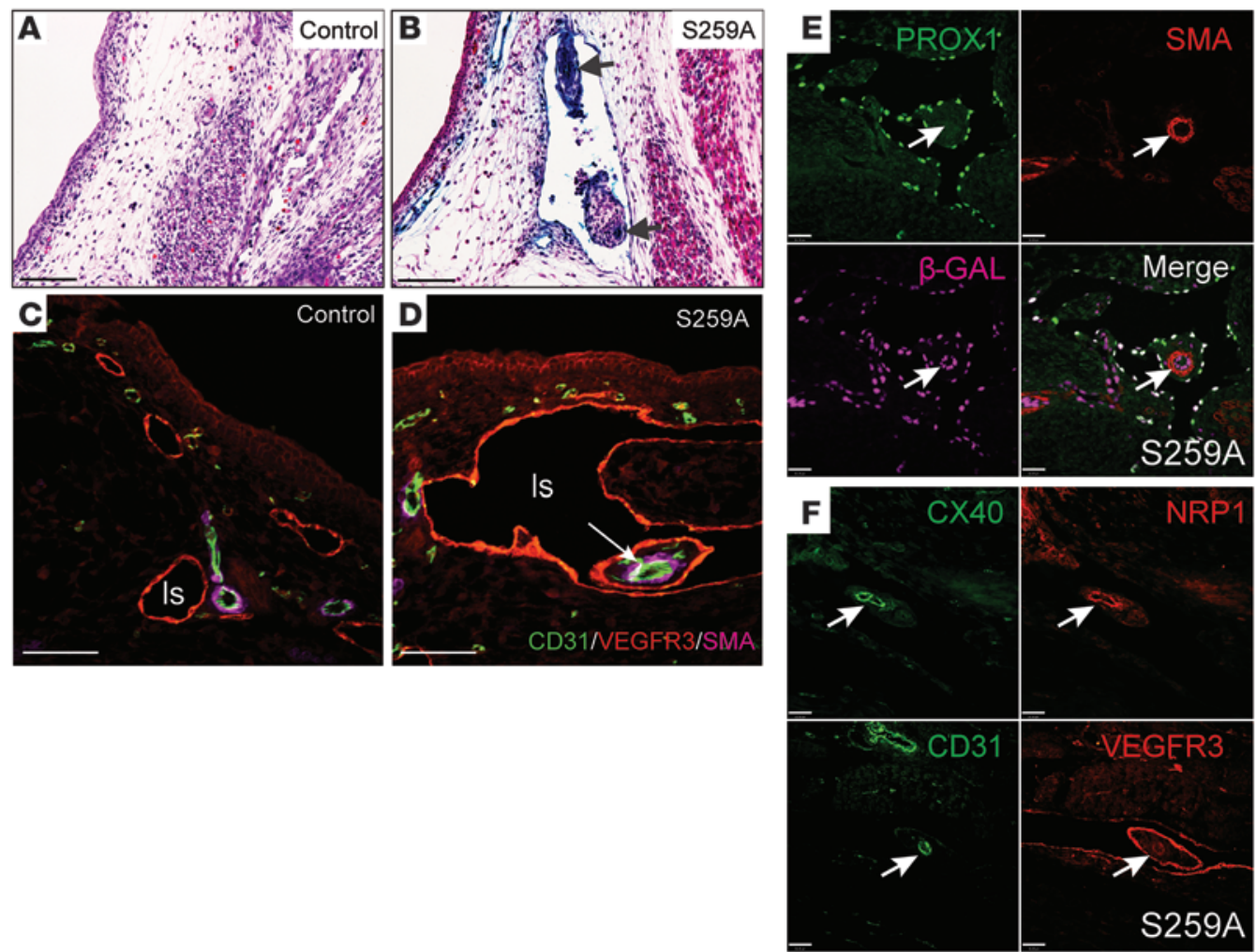

\section{Figure 3}

RAF1S259A mice develop lymphangiectasia. X-gal staining ( $\mathbf{A}$ and $\mathbf{B}$ ) and immunofluorescence staining of E14.5 embryo sections (C-F) revealed malformed lymphatic vessels in S259A embryos. Note that small vessels inside the enlarged lymphatics were stained with $\beta$-gal (B and $E$, arrows), SMA (D and E, arrows), CD31, connexin 40, and NRP1 (F, arrows), but not VEGFR3 (D) and PROX1 (E). Scale bars: $80 \mu \mathrm{m}$ (A-D); $36 \mu \mathrm{m}$ (E and F). Is, lymph sac. phatic vessels (Figure 2C) in S259A embryos compared with control littermates. VEGFR3 and PROX1 immunofluorescence staining confirmed the lymphatic nature of these vessels (Figure 2D). Lumen sizes of subcutaneous lymphatic vessels in E14.5 S259A embryos were on average 5-fold larger than those of the control littermates (Figure 2E), with approximately $35 \%$ of the lymphatic vessels in S259A embryos having a lumen size greater than $5000 \mu \mathrm{m}^{2}$, while in control embryos, no lymphatic vessels of such size were observed (Figure 2F). Moreover, while greater than $50 \%$ of the subcutaneous lymphatic vessels in control embryos had lumen sizes smaller than $500 \mu \mathrm{m}^{2}$, less than $2 \%$ of the lymphatic vessels in the S259A embryos were that small. In agreement with the lumen size data, the diameters of the skin lymphatic vessels in E14.5 S259A embryos were on average twice as large as those of the control littermates (Figure 2, G and $\mathrm{H}$ ). At the same time, there were no major differences in the size and/or diameter of blood vessels in S259A embryos.

Besides gross enlargement, another common feature of the lymphatics in S259A embryos was the presence of connexin 40-positive (CX40), neuropilin 1-positive (NRP1), smooth muscle actin-positive (SMA), and VEGFR3- and PROX1-negative arteries surrounded by dilated lymphatics in skin, a feature not seen in control mice and a pathognomonic finding in lymphangiectasia syndromes (Figure 3) (5). Taken together, these results demonstrate that S259A mice develop lymphangiectasia reminiscent of the lymphatic phenotypes in Noonan syndrome and related disorders.

RAF1 ${ }^{5259 A}$ induction of PROX1 expression. Massive lymphatic enlargement in S259A embryos suggests either increased LEC proliferation or increased fate specification. To address the former possibility, the fraction of $\mathrm{Ki} 67^{+} / \mathrm{PROX} 1^{+}$cells among all PROX $1^{+}$ cells was determined in both E12.5 (Supplemental Figure 2, A-C) and E14.5 (Supplemental Figure 2, D-F) embryos. No signifi- cant differences in the percentage of proliferating LECs in S259A embryos versus littermate controls were observed (Supplemental Figure 2, B and E). However, the total number of PROX $1^{+}$cells was twice as high in the S259A embryos compared with that in the control embryos (Supplemental Figure 2, C and F), suggesting increased lymphatic fate specification.

To address this possibility, we next examined the kinetics of PROX1 expression, given its critical role in lymphatic fate specification (27-30). At E10.5, a stage when PROX1 expression is initiated by SOX18 specifically in the dorsolateral portion of the cardinal veins, only a few of the PROX1-positive cells were observed in wild-type embryos (Figure 4, A and B). However, in S259A embryos, PROX1 was detected not only in cardinal veins, but in dorsal aortae as well (Figure 4C). In addition, many PROX1-positive cells were seen migrating out of cardinal veins (Figure 4A). The same phenomenon was also observed in E11.5 embryos (Figure 5A). At E12.5, in the wild-type embryos, PROX1 was evident only in lymphatic sacs, with almost no expression detectable in cardinal veins (Figure 4, A and D). In contrast, PROX1 expression persisted in cardinal veins, dorsal aortae, and lymphatic sacs in S259A embryos (Figure 4E).

By E14.5, PROX1 was still detectable in the cardinal veins of S259A embryos, although at a lower level compared with that in jugular lymphatic sacs (Supplemental Figure 3). Surprisingly, despite the presence of PROX1, no obvious expression of other lymphatic markers, such as VEGFR3 (Figure 4A or Supplemental Figure 3) or podoplanin (data not shown), was observed in blood ECs within major arteries and veins of S259A embryos at E12.5 or E14.5. In contrast, all of the PROX1-positive cells migrating dorsolaterally from the jugular vein expressed VEGFR3.

Lymphatic specification in mice is thought to begin at E11.5 when PROX1-positive cells delaminate from cardinal veins and 
A

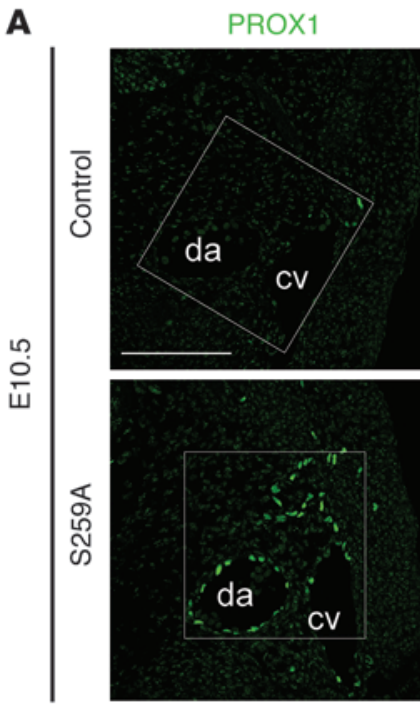

$\mid$

$\frac{1}{\amalg}$
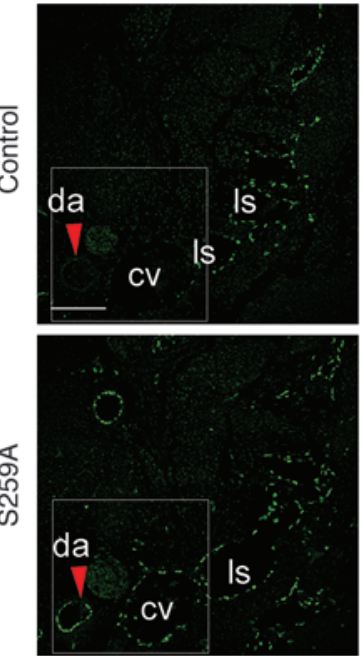

$\beta$-gal
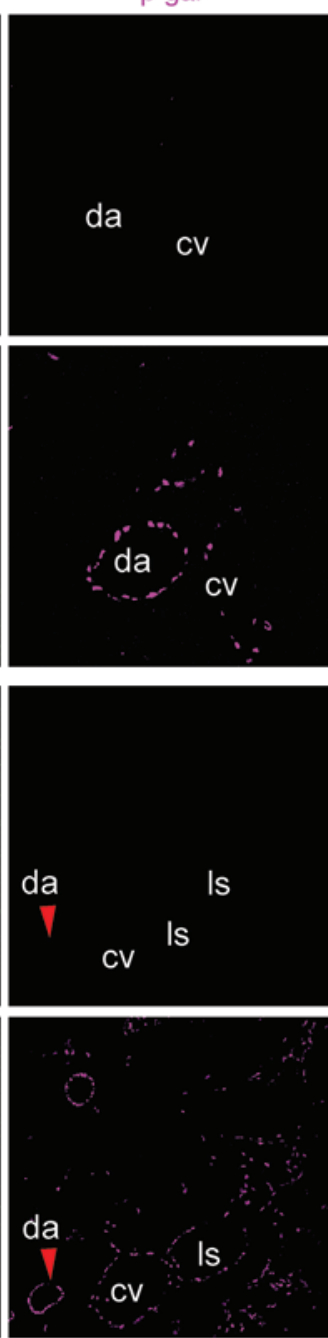

VEGFR3
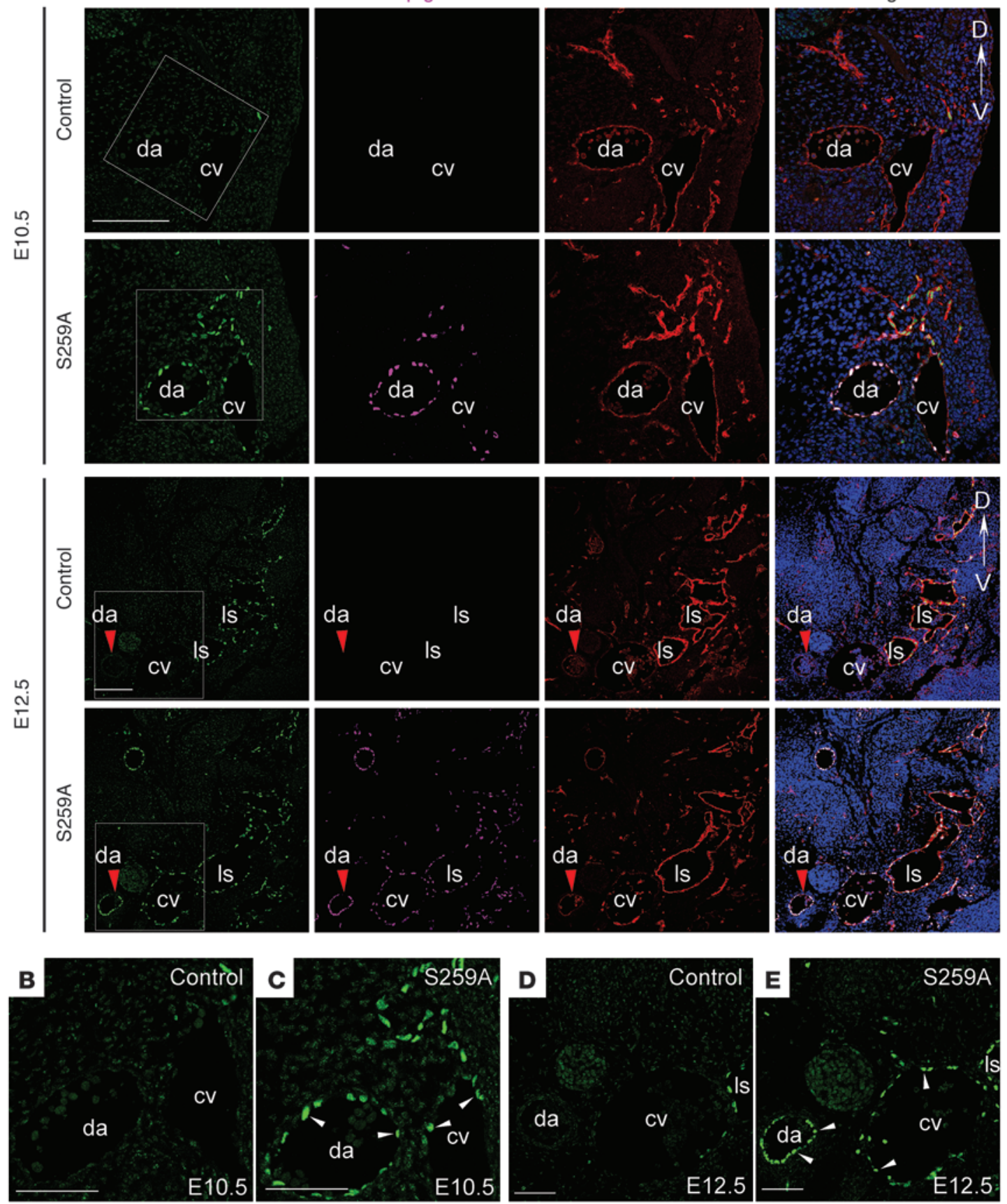

Figure 4

RAF1 ${ }^{\text {S259A }}$ induces PROX1 expression in blood ECs. (A) Immunofluorescence staining of PROX1 (green), $\beta$-gal (magenta), and VEGFR3 (red) in E10.5 and E12.5 embryo sections. Nuclei were stained with DAPI (blue). (B-E) Higher-magnification images of areas highlighted with squares in (A). PROX1-positive cells in the dorsal aorta and the cardinal vein are indicated with white arrowheads. Red arrowheads point to the dorsal aorta. Scale bars: $200 \mu \mathrm{m}$ (A); $100 \mu \mathrm{m}$ (B-E). da, dorsal aorta; D, dorsal; V, ventral.

migrate dorsolaterally to form jugular lymphatic sacs. Quantitative analysis showed that the number of these migrating cells had almost doubled in S259A embryos compared with control embryos at both E11.5 and E12.5 (Figure 5B). In addition, both the total number of PROX $1^{+}$cells and the number of PROX $1^{+}$ cells in cardinal veins were significantly higher in S259A embryos (Figure 5B).
To confirm these results in vitro, HUVECs were transduced with an adenovirus encoding RAF1 $1^{\mathrm{S} 259 \mathrm{~A}}$ (Ad-RAF1 ${ }^{S 259 A}$ ). This resulted in increased expression of PROX1 and various lymphatic genes encoding VEGFR3, LYVE1, and PDPN (Figure 5C).

SOX18 is upregulated in S259A embryos. During early embryonic development, lymphatic fate specification is initiated by SOX18, which directly controls Prox1 gene expression. To study 
A
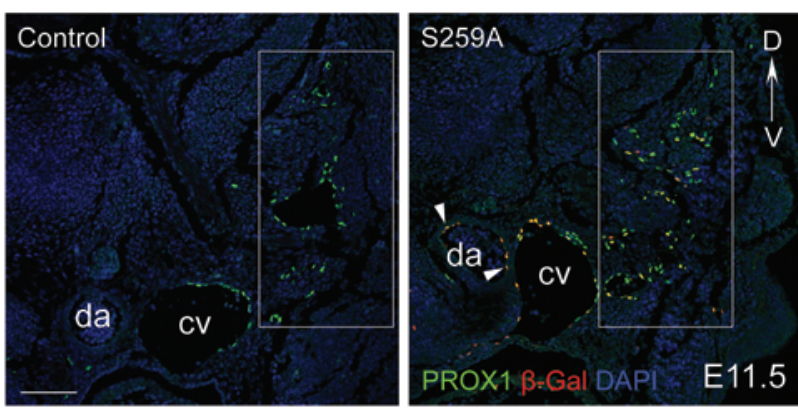

B PROX $1^{+}$cells outside $\mathrm{CV}$

PROX $1^{+}$cells in CV
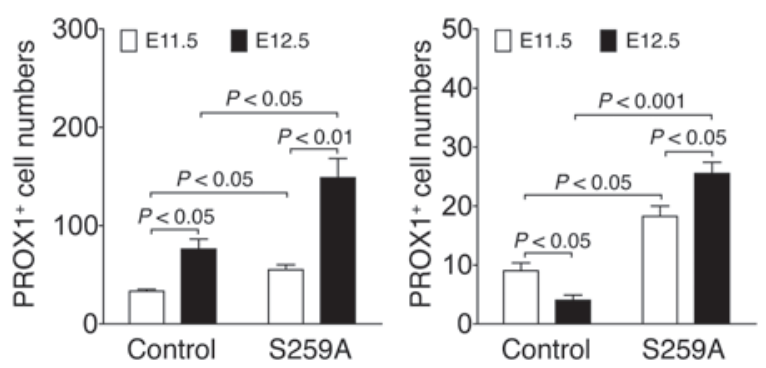

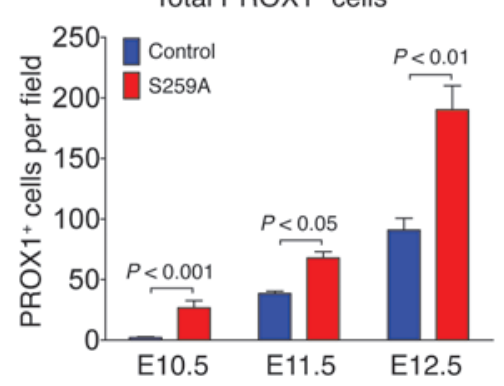

\section{Total PROX $1^{+}$cells}

\section{Figure 5}

Blood endothelial RAF1S259A expression induces lymphatic EC fate specification. (A) Immunofluorescence staining of PROX1 (green) and $\beta$-gal (red) in E11.5 embryo sections revealed more migrating $\mathrm{PROX} 1^{+}$cells from the cardinal vein (highlighted within rectangle insets). White arrowheads indicate PROX $1^{+}$cells in the dorsal aorta. Scale bar: $200 \mu \mathrm{m}$. (B) Quantification of PROX1+ cells. PROX $1^{+}$cells outside the cardinal vein were designated as those that dorsolaterally migrated out of veins and excludes those cells in the dorsal aorta. An example of "PROX $1^{+}$cells outside CV" is shown in (B) and highlighted in the rectangle insets. Control, $n=4$ embryos; S259A, $n=4$ embryos. Mean \pm SEM. (C) qPCR analysis of lymphatic marker gene expression in lentivirus-transduced HUVECs. Mean \pm SEM; $n=3$.
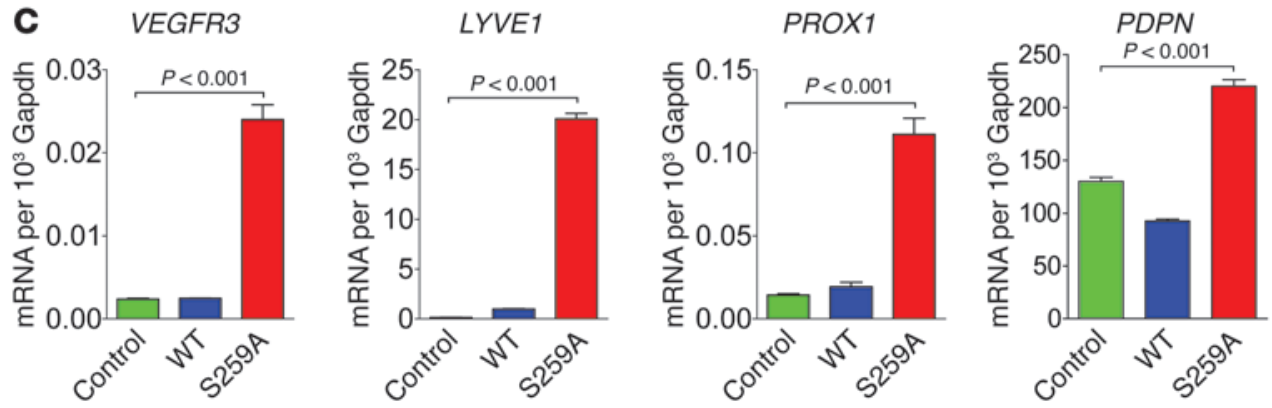

the mechanism of RAF1 $1^{\mathrm{S} 259 \mathrm{~A}}$-dependent induction of lymphatic fate, we investigated its effect on SOX18 expression in vivo using immunofluorescence staining. Wild-type embryos displayed low levels of SOX18 expression at various developmental stages from E10.5 to E12.5. Conversely, strong SOX18 expression was observed in all of the $\beta$-gal-positive cells in S259A embryos (Figure 6A or Supplemental Figure 4). During normal LEC fate induction, SOX18 is only transiently expressed in cardinal vein ECs (prior to induction of PROX1 expression), and its expression is shut down after E11.5 and shut off by E14.5 (17). In contrast to the wild-type mice, S259A embryos demonstrated stronger SOX18 expression in the blood vasculature including both arteries and veins at E10.5, with the expression persisting through E14.5, both in blood and lymphatic vessels (Figure 6, B-D, and Supplemental Figure 4), suggesting that ongoing RAF/ERK activation due to RAF1 $1^{\mathrm{S} 259 \mathrm{~A}}$ expression can overcome normal downregulation of SOX18 expression.

To verify these results, we measured SOX18, SOX17, and SOX7 expression in HUVECs transduced with Ad-RAF1 ${ }^{S 259 A}$. In agreement with the in vivo data, expression of RAF1 $1^{S 259 A}$, but not a wild-type $R A F 1$, induced a significant increase in SOX18 and SOX17 levels, while $S O X 7$ remained unchanged (Figure 7, A and B). To confirm Sox18 induction by RAF1 $5259 \mathrm{~A}$ in vivo, we examined its expression in primary ECs purified from E12.5 S259A embryos. In agreement with in vitro results, Sox18 levels were significantly higher compared with ECs isolated from control littermates (Figure 7C).

While COUP-TFII has also been shown to be required for the initiation and early maintenance of PROX1 expression in LECs (31), endothelial $R A F 1^{S 259 A}$ expression had no significant effect on COUPTFII levels in vitro or in vivo (Supplemental Figure 5). PROX1 has been shown to control the number of LEC progenitors (32) and the budding out of these progenitors from the cardinal vein (33). Thus, a persistent increase in SOX18 and PROX1 expression in cardinal veins of S259A embryos can be expected to result in prolonged venous EC-to-LEC differentiation and a continuous migration of PROX1-positive LEC progenitors to lymph sacs. This indeed was the case, as shown in Figure 4 and Figure 5, A and B, where there was a larger number of migrating PROX1-positive cells from cardinal veins in S259A embryos. As a result, by E14.5, jugular veins in S259A embryos were significantly smaller than in littermate controls, while the carotid artery size was largely unchanged (Figure 7, D-F).

RAF1-AKT crosstalk regulates lymphatic endothelial fate specification. The Ser259 to Ala259 mutation of RAF1 decouples the RAF1/AKT crosstalk and thus renders RAF1 resistant to inhibition by PI3K/ AKT signaling. This results in simultaneous activation of both RAF1/ERK and PI3K/AKT signaling pathways. In primary mouse lung ECs isolated from S259A mice, RAF1 ${ }^{\text {S259A }}$ expression resulted in a $45 \%$ increase in baseline phospho-ERK level (Figure 8A), 
A
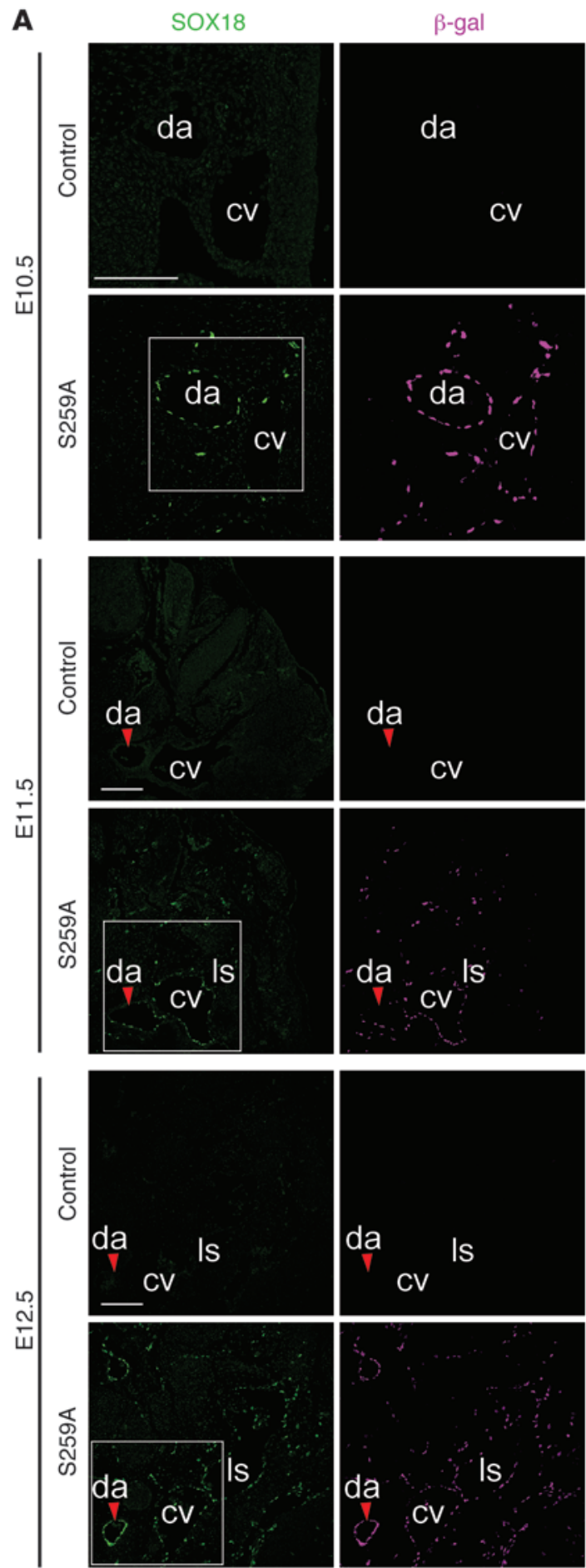
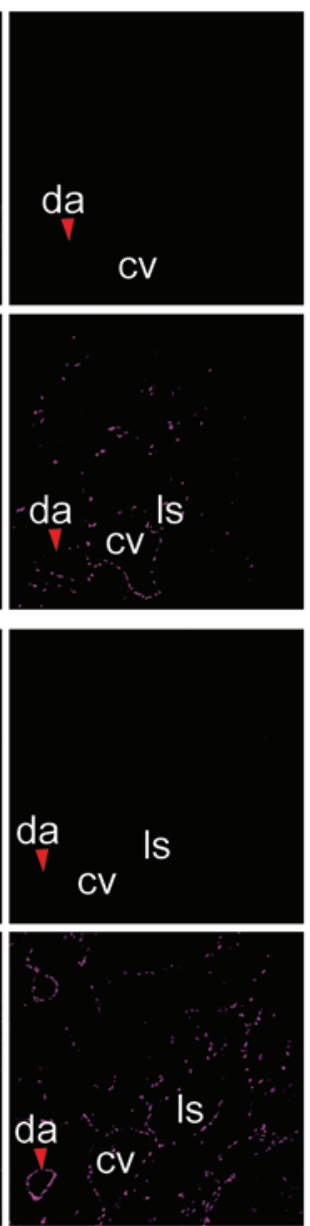

VEGFR3
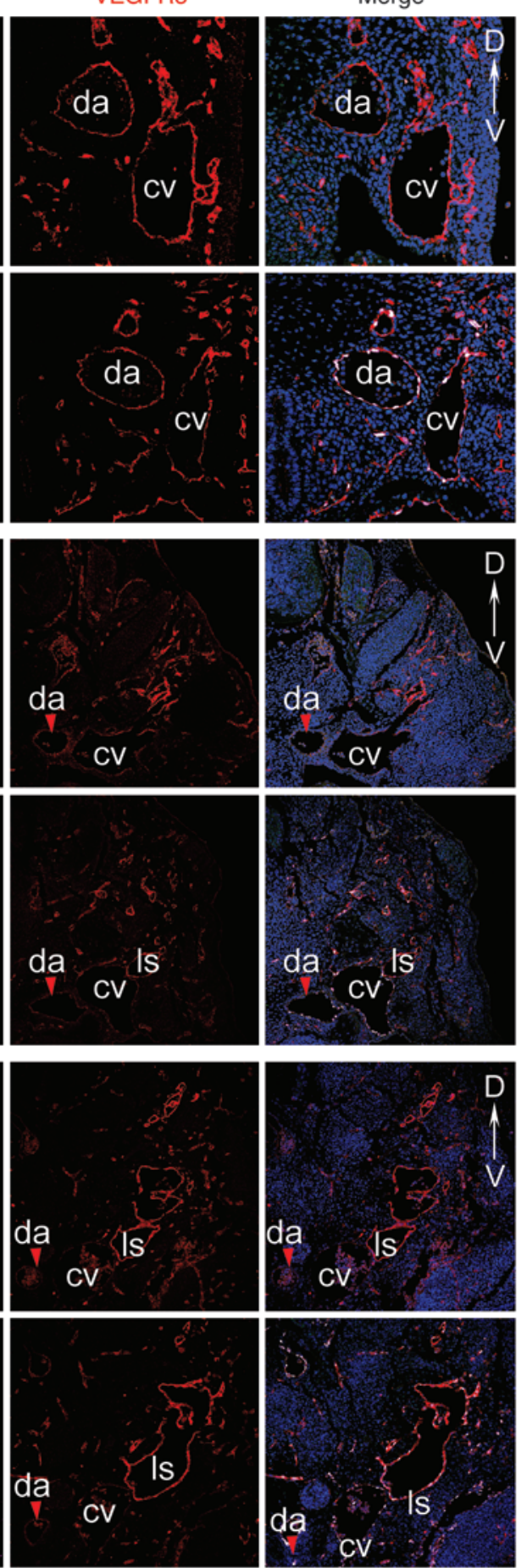
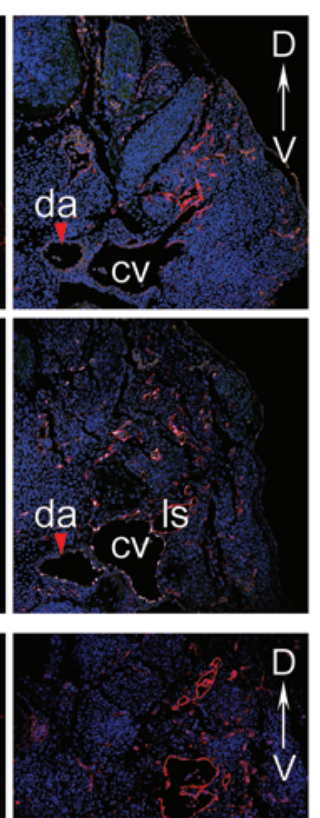

da
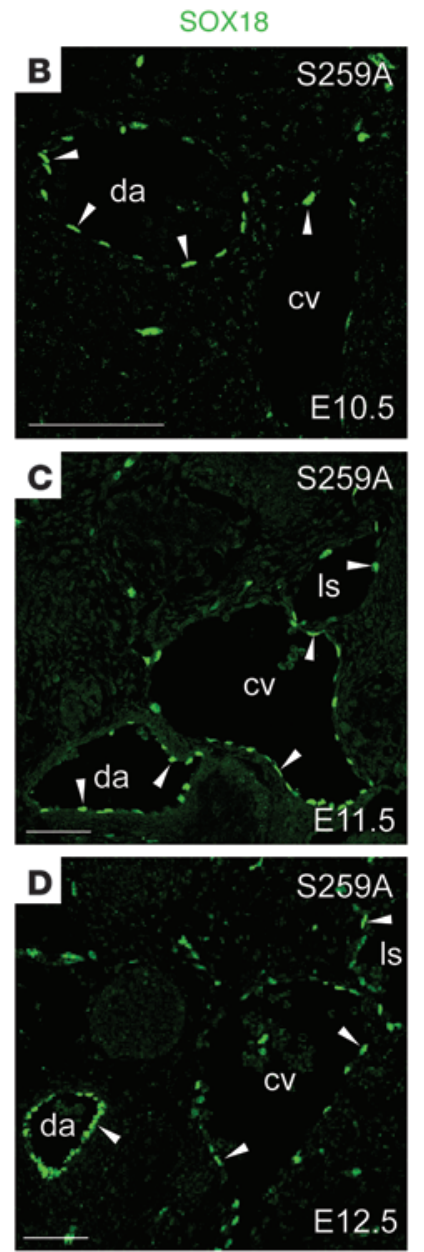

E12.5

Figure 6

SOX18 expression is upregulated in RAF1S259A embryos. (A) Immunofluorescence staining of SOX18 (green), $\beta$-gal (magenta), and VEGFR3 (red) in E10.5, E11.5, and E12.5 embryo sections. Nuclei were stained with DAPI (blue). (B-D) Higher-magnification images of areas highlighted with squares in (A). SOX18-positive cells in the dorsal aorta and the cardinal vein are indicated with white arrowheads. Red arrowheads in (A) point to the dorsal aorta. Scale bar: $200 \mu \mathrm{m}$.

which is comparable to the ERK activation seen in another Noonan syndrome mouse model (34). The ongoing ERK activation in S259A embryos was demonstrated by anti-pERK1/2 staining of $\beta$-gal-positive ECs (Figure 8B).
To determine the specific roles of ERK and AKT signaling in lymphatic fate specification, we next studied the effect of selective inhibition of either pathway's signaling on SOX18, PROX1, VEGFR3, and LYVE1 expression in HUVECs in vitro. Shutdown 

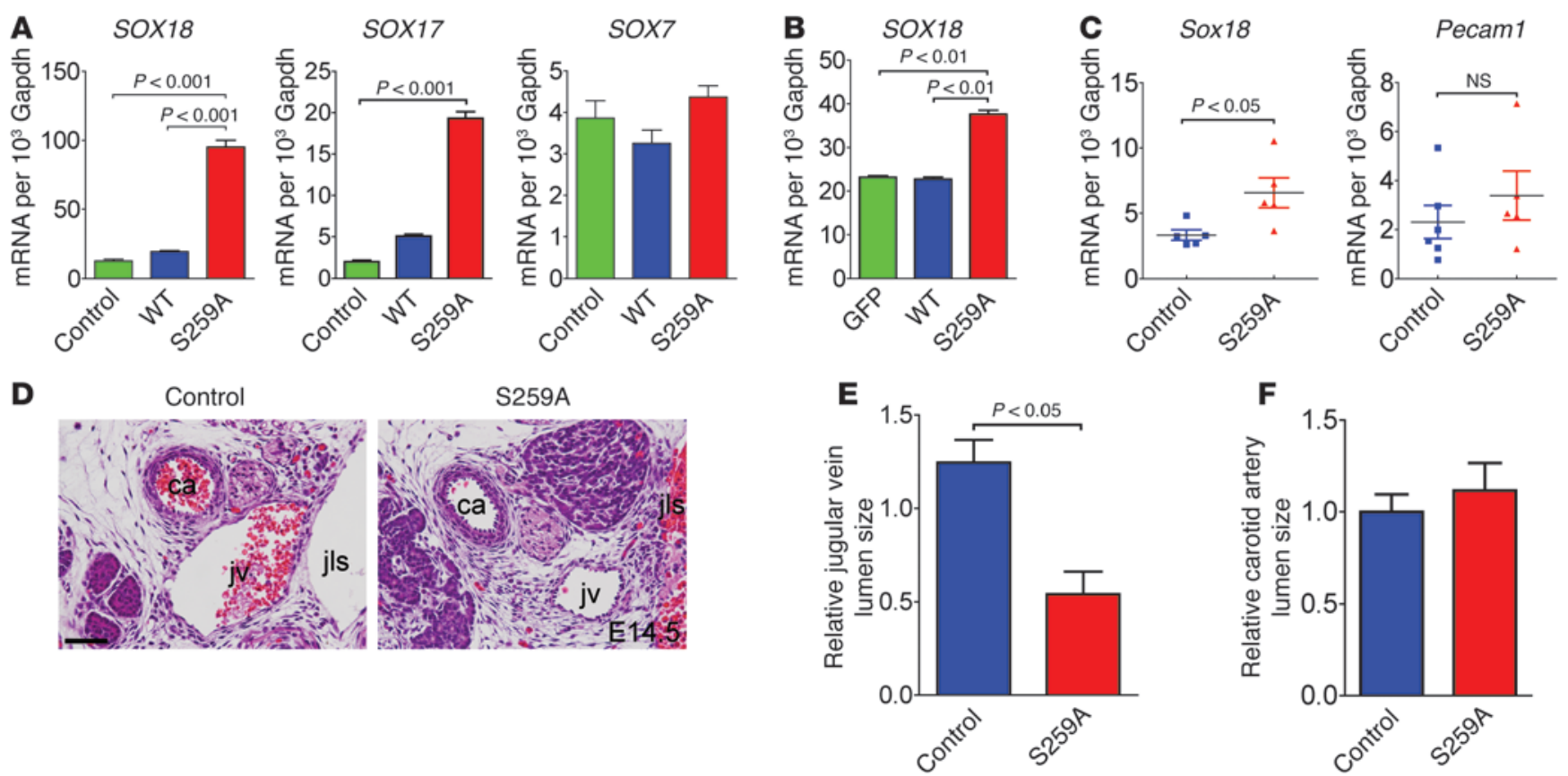

Figure 7

RAF1S259A induces Sox18 expression. (A) qPCR of SOX18, SOX17, and SOX7 in HUVECs transduced with null (control), wild-type RAF1 (WT), or $R A F 1^{\text {S259A }}$ (S259A) lentiviruses. Mean \pm SEM; $n=3$. (B) qPCR analysis of SOX18 expression of HDLECs infected with adenoviruses expressing GFP, wild-type RAF1 (WT), or RAF1S259A (S259A) constructs. Mean \pm SEM; $n=3$. (C) qPCR analysis of Sox18 and Pecam1 expression in primary ECs isolated from E12.5 embryos. Mean $\pm \mathrm{SEM} ; n=5$ embryos. (D) H\&E staining of E14.5 embryos demonstrating larger jugular lymphatic sacs, smaller jugular veins, and normal-sized carotid arteries in S259A compared with control embryos. Scale bar: $28 \mu \mathrm{m}$. (E and F) Quantification of lumen sizes of the jugular vein (E) and the carotid artery $(\mathbf{F})$ at the indicated positions (heart level) as shown in (D). The values were then normalized to that of carotid arteries and averaged to represent the mean number for each embryo. The average carotid artery lumen size in control embryos was designated as 1. Control, $n=5$ embryos; S259A, $n=3$ embryos. Mean \pm SEM. ca, carotid artery.

of the RAF1/MEK/ERK pathway by the MEK inhibitor U0126 blocked induction of all 4 genes by RAF1 ${ }^{\mathrm{S} 259 \mathrm{~A}}$ (Figure $8 \mathrm{C}$ ). In contrast, inhibition of PI3K/AKT signaling led to a slight increase in SOX18 expression, likely due to baseline AKT suppression of RAF1 activation. At the same time, expression of PROX1, VEGFR3, and LYVE1 (Figure 8C) was significantly reduced by PI3K inhibition. In agreement with these findings, inhibition of mTOR by rapamycin or direct inhibition of AKT activation by AKT inhibitor VIII also significantly inhibited PROX1 and COUPTFII expression, while slightly inducing SOX18 expression (Figure $8 \mathrm{D})$. These results suggest that SOX18 expression is exclusively dependent on the RAF1/MEK/ERK signaling pathway, while PROX1, VEGFR3, and LYVE1 expression requires both MEK/ERK and PI3K/AKT signaling inputs. Interestingly, in RAF $1^{\text {S259A-over- }}$ expressing ECs, the crosstalk between AKT and ERK appears not to be completely disrupted. Inhibiting endogenous AKT pathways via inhibitors still promotes SOX18 expression in $R A F^{S 259 A_{-}}$ expressing cells.

We next verified that RAF $1^{\mathrm{S} 259 \mathrm{~A}}$ expression induces the lymphatic phenotype via activation of ERK signaling, and not in an ERK-independent fashion. To this end, two previously described constitutively active MEK/ERK constructs in adenoviral vectors a nuclear-localizing construct (Ad-ME-LA) and a construct lacking the nuclear localization signal (Ad-ME) (9) - were used to transduce HUVECs in culture. Expression of the $M E-L A$, but not the $M E$, construct induced expression of SOX18, PROX1, VEGFR3, LYVE1, and PDPN in HUVECs (Figure 8E).
Suppression of excessive ERK activation rescues the lymphatic phenotype in S259A embryos. Given the essential role of ERK signaling in SOX18 and PROX1 induction, we next determined whether inhibition of MEK/ERK signaling can rescue the lymphatic phenotype in S259A embryos. Two i.p. injections of the MEK inhibitor U0126 into pregnant mice at E10.5 and E11.5, the time when the lymphatic network is forming, led to a significant decrease in lymphatic sac formation in S259A embryos at E12.5 (Figure 9, $A$ and $B$ ). In particular, the number of SOX18- and PROX1positive cells decreased by approximately $40 \%$ in U0126-treated embryos compared with untreated S259A embryos (Figure 9, A and B, and Supplemental Figure 6). Furthermore, there was a significant inhibition of Sox18, Vegfr3, Lyve1, and Pdpn expression in primary mouse embryonic ECs (Figure 10A). Finally, i.p. injection of U0126 into pregnant mice at E13.5, the time when the lymphatic network is forming in the skin of mouse embryos, led to a significant decrease in skin lymphatic vessel size in S259A embryos 24 hours later (Figure 10, B and C).

\section{Discussion}

The data presented in this study suggest a model in which RAF1/ MEK/ERK signaling induces LEC fate specification and lymphatic vessel development by controlling SOX18 expression (Supplemental Figure 7). ERK activation, regulated by RAF1-AKT crosstalk, controls SOX18 and PROX1 expression in venous ECs, which leads to delamination of these LEC progenitors and eventually to full LEC fate specification. In Noonan syndrome and related 
A

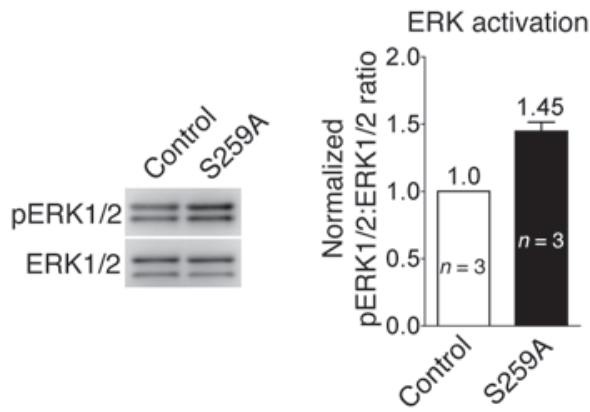

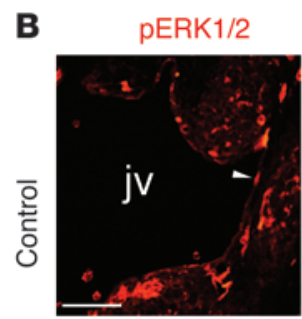
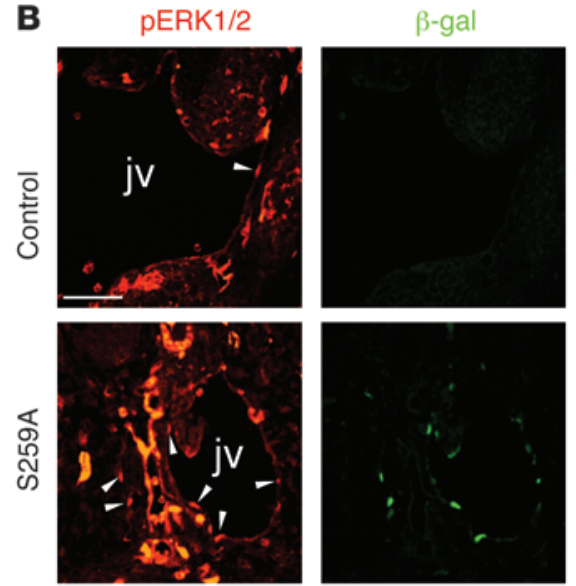

VEGFR3

PROX1
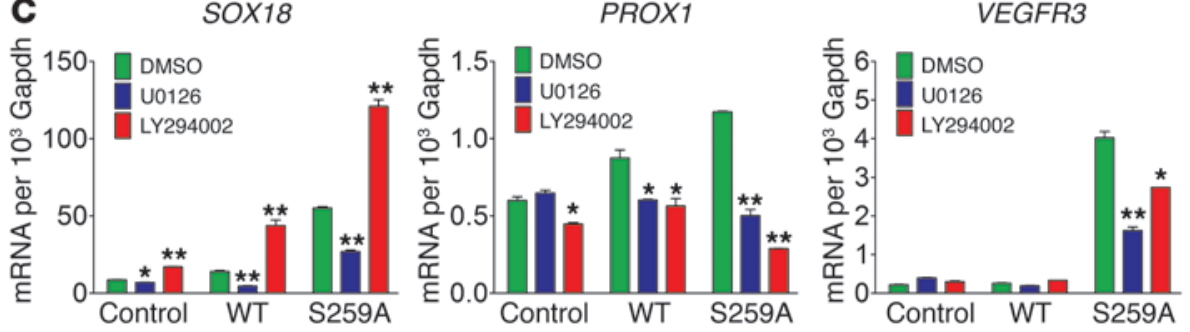

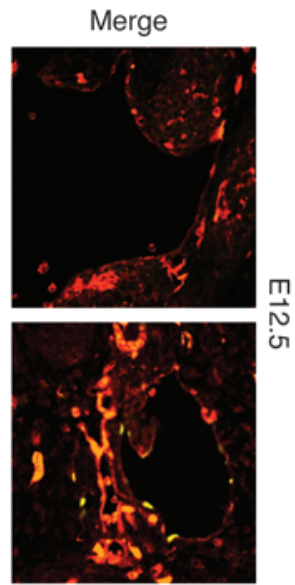

LYVE1

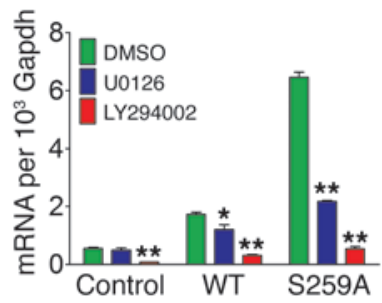

D

SOX18

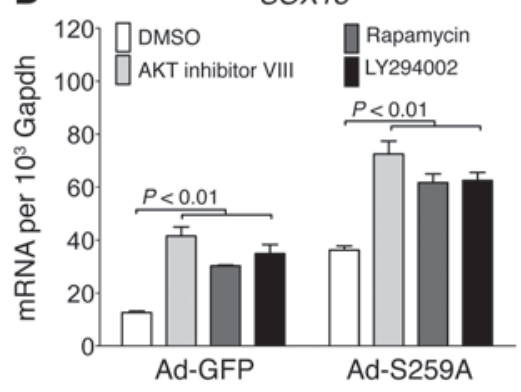

PROX1
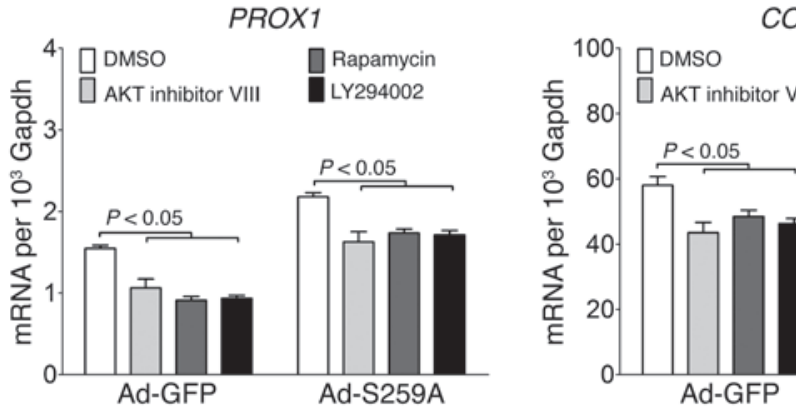

COUP-TFII
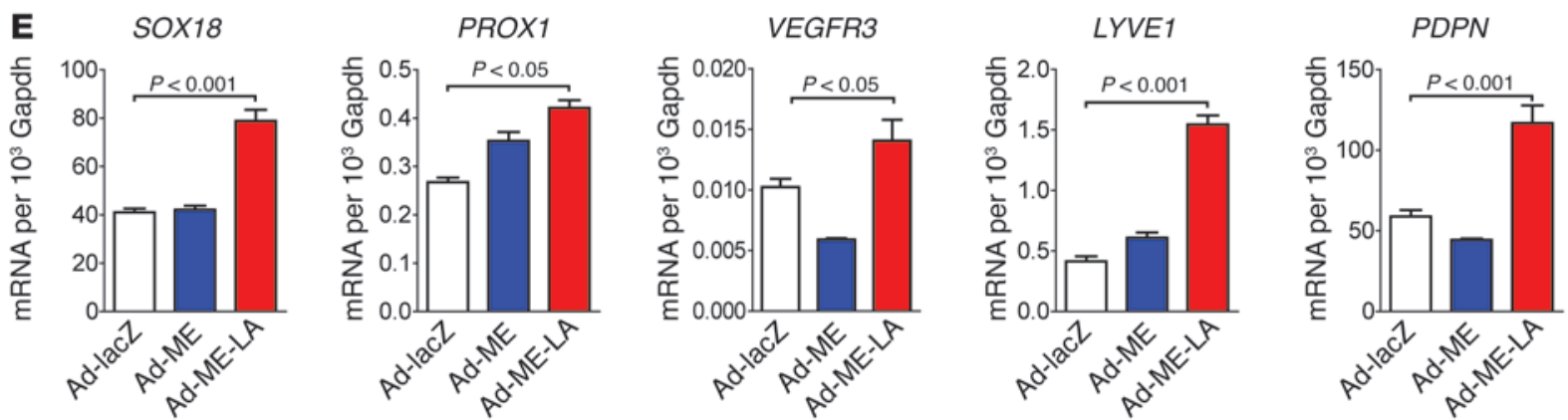

Figure 8

RAF1-AKT crosstalk regulates lymphatic endothelial fate specification by controlling ERK activation. (A) Western blot shows that ERK is activated in S259A primary mouse lung ECs (left panel). ERK activity was quantified by densitometry and is represented as the ratio of $p E R K 1 / 2$ to total ERK1/2 (right panel). Data represent the mean \pm SEM of 3 independent experiments. (B) Immunofluorescence staining showing higher $p E R K 1 / 2$ (red, arrowheads) in $\beta$-gal-positive (green) ECs of E12.5 S259A embryos. Scale bar: $100 \mu \mathrm{m}$. (C) Effect of MEK and PI3K inhibition on lymphatic gene expression. HUVECs transduced with control, wild-type RAF1, or RAF1S259A lentiviruses were treated with DMSO, MEK inhibitor U0126 $(10 \mu \mathrm{M})$, or PI3K inhibitor LY294002 $(10 \mu \mathrm{M})$ for 24 hours. SOX18, PROX1, VEGFR3, and LYVE1 expression was assessed by qPCR. Data represent the mean \pm SEM of 3 independent experiments. ${ }^{*} P<0.05 ;{ }^{* *} P<0.01$. (D) Effect of $m T O R$ and AKT inhibition on SOX18, $P R O X 1$, and COUP-TFIl expression. HUVECs transduced with GFP or RAF1 $2259 A$ adenoviruses were treated with DMSO, rapamycin (10 $\mu \mathrm{M}), \mathrm{AKT}$ inhibitor VIII $(10 \mu \mathrm{M})$, or LY294002 (10 $\mu \mathrm{M})$ for 24 hours. SOX18, PROX1, and COUP-TFIl expression was assessed by qPCR. Data represent the mean \pm SEM of 3 independent experiments. (E) Constitutive active ERK is able to induce lymphatic genes. HUVECs were transduced with adenovirus expressing lacZ, cytosolic localized constitutive active ERK (ME), or nuclear localized constitutive active ERK (ME-LA). SOX18, PROX1, VEGFR3, $\angle Y V E 1$, and $P D P N$ expression was assessed by qPCR. Data represent the mean \pm SEM of 3 independent experiments. jv, jugular vein. 
A
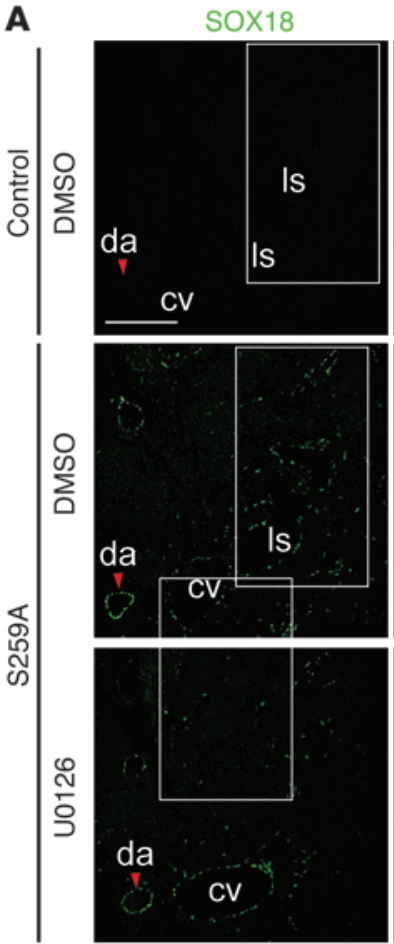

B

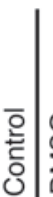

$\mid$

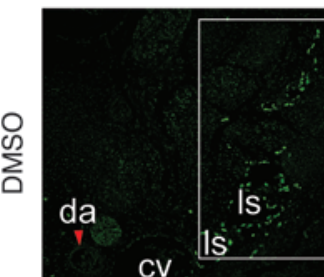

CV

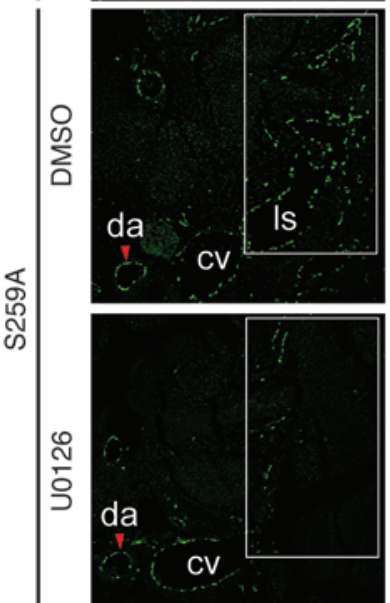

$\beta$-gal
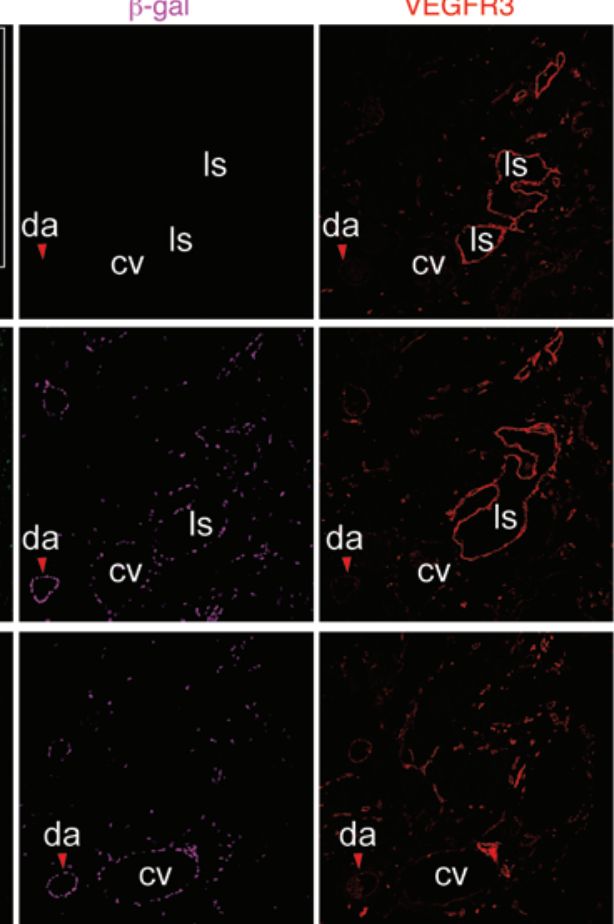

$\beta$-gal
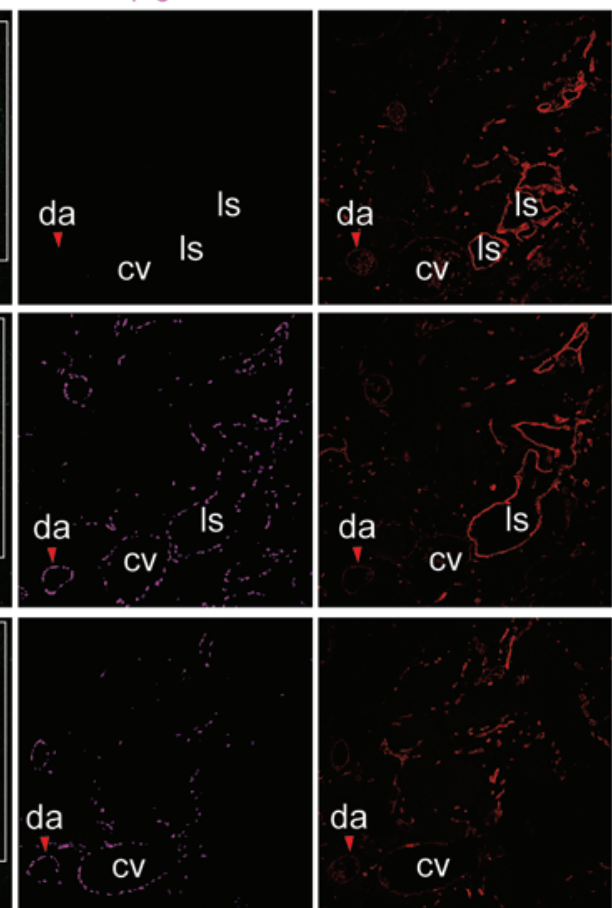

Merge
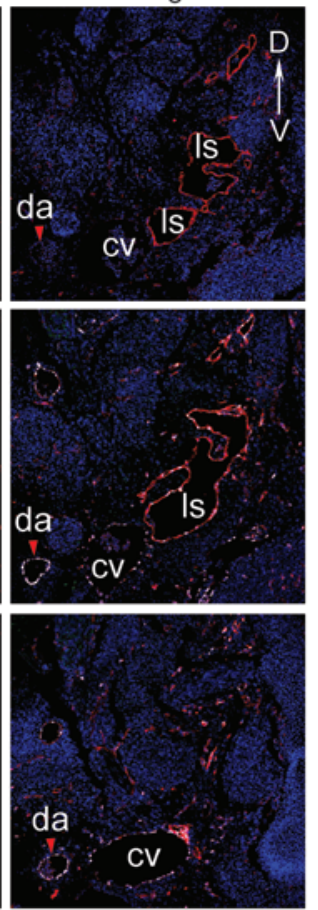

Merge
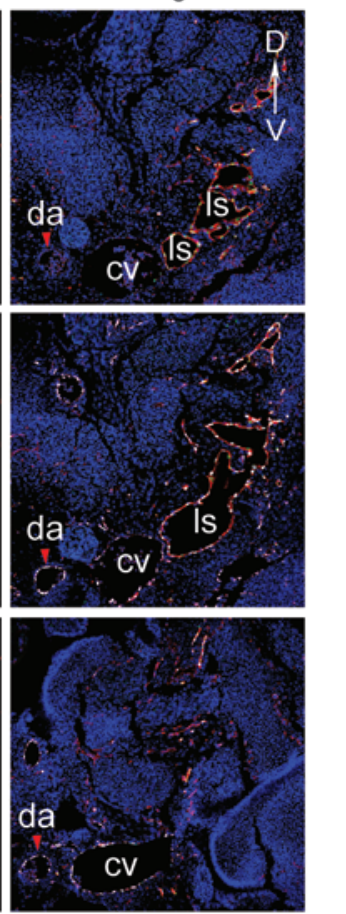
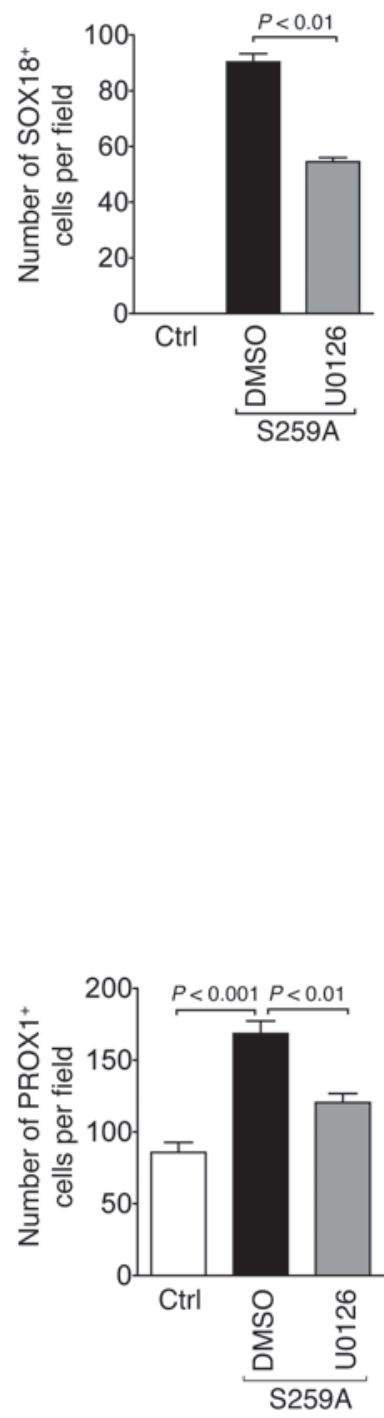

Figure 9

Inhibition of ERK signaling reduces lymphatic EC specification. (A and B) Immunofluorescence staining of SOX18 (A) or PROX1 (B) (green), $\beta$-gal (magenta), and VEGFR3 (red) of DMSO or U0126-treated E12.5 embryo sections. Nuclei were stained with DAPI (blue). Red arrowheads point to the dorsal aorta (E12.5). Scale bars: $200 \mu \mathrm{m}$. Quantification of SOX18+ (A, right panel) and PROX1+ (B, right panel) cells was performed by counting SOX $18^{+}$or PROX $1^{+}$cells highlighted in the inset rectangles at the indicated positions (heart), as shown in (A and $\mathbf{B}$, left panels). Control, $n=4$ embryos; S259A, $n=4$ embryos. Mean \pm SEM. 
A

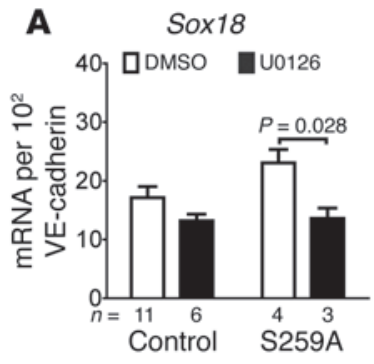

Lyve1

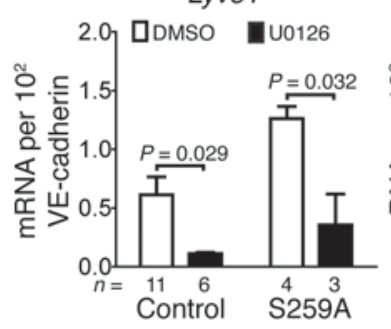

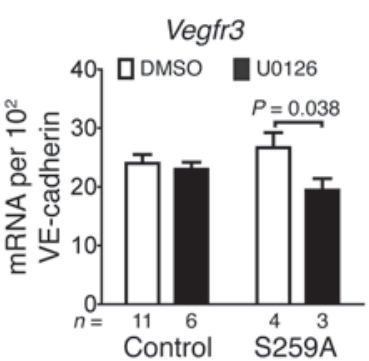

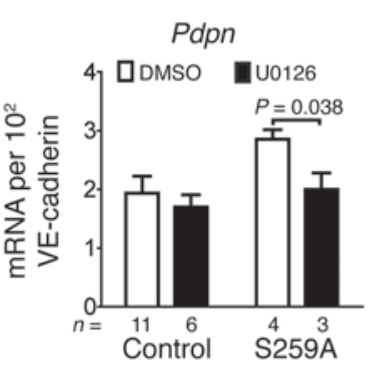

B
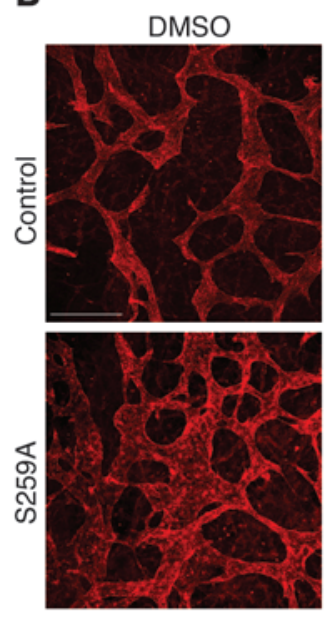
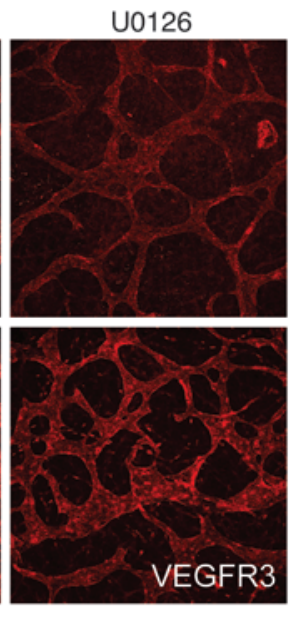

C

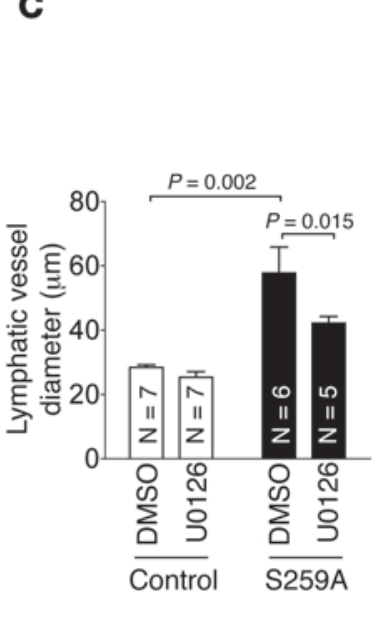

\section{Figure 10}

Inhibition of ERK signaling rescues the lymphatic phenotype in RAF1S259A embryos. (A) qPCR analysis of Sox18, Vegfr3, Lyve1, and Pdpn expression in primary ECs isolated from DMSO or U0126-treated E12.5 embryos. Data represent the mean \pm SEM. Control: DMSO, $n=11$ embryos; U0126, $n=6$ embryos; S259A: DMSO, $n=4$ embryos; U0126, $n=3$ embryos. (B) Whole-mount staining of dorsal skin vasculature of E14.5 embryos treated with MEK inhibitor U0126 or DMSO. Scale bar: $200 \mu \mathrm{m}$. (C) Quantitative analysis of lymphatic vessel diameters based on VEGFR3 staining shown in (B). Mean \pm SEM. Control: DMSO, $n=7$ embryos; U0126, $n=7$ embryos; S259A: DMSO, $n=6$ embryos; U0126, $n=5$ embryos.

"RASopathy" patients, excessive ERK signaling leads to a persistent induction of SOX18 and PROX1 in venous ECs, resulting in increased transition of these venous ECs to a lymphatic fate, leading, in turn, to increased outmigration of these newly specified LECs to the forming lymphatic sacs. The sacs are greatly increased in size and give rise to disproportionally large lymphatics, thus leading to lymphangiectasia. At the same time, with the exception of the right jugular sac in about $50 \%$ of the embryos where some hemorrhage was observed, no blood was observed anywhere else in any of the lymphatics, suggesting complete separation of the venous and lymphatic circulations.

The morphologic features observed in $R A F 1^{S 259 A}$ transgenic mice include large, irregularly shaped lymphatic vessels wrapping around arterioles and small arteries, a finding pathognomonic to patients with Noonan syndrome and extensive edema. Gainof-function RAF1 or ERK mutations are observed in 3\%-17\% of Noonan syndrome cases $(11,26,35)$, and the increase in ERK signaling observed in these patients is of the same order of magnitude (less than 2-fold) as that seen in this study (1.45-fold increase). Thus, the lymphangiectasia observed in these patients is likely attributable to the effect of excessive ERK signaling on lymphatic fate specification, as suggested by our study.

VEGF-A (a principal VEGF in arterial and venous ECs) and VEGF-C (the key VEGF in lymphatic ECs), via their respective receptors VEGFR2 and VEGFR3, activate several signaling cascades including PI3K/AKT and RAS/RAF/ERK. Until recently, no interactions between these two signaling cascades have been appreciated. We have previously demonstrated that under normal conditions in the adult vasculature, AKT inhibits ERK signaling via phosphorylation of RAF1, and that inhibition of PI3K leads to ERK activation (9). The importance of PI3K/ERK interaction is further suggested by studies demonstrating that mice lacking the
Pik3r1 regulatory subunit display defects in lymphatic remodeling and maturation, while lymphatic vessels display upregulation of biliary EC markers such as endoglin (36).

In the present study, we examined the role of PI3K/AKT-RAF1/ MEK/ERK crosstalk in the developing vasculature using an RAF1 mutant insensitive to AKT phosphorylation. Since the promoter construct used in these studies results in activation of the mutant construct expression at approximately E9.5, the time of lymphatic fate specification, we concentrated on the effect of this mutation on lymphangiogenesis. Decoupling of PI3K and ERK pathways in vitro resulted in activation of ERK, even in the absence of VEGF (or other growth factor) stimulation, demonstrating that the former tonically suppresses the latter. In vivo, this resulted in activation of Sox18 expression in cardinal veins and led to induction of PROX1 expression which, in turn, initiated lymphatic fate specification and lymphangiogenesis. These results strongly argue that ERK controls SOX18 expression and that transient activation of ERK signaling, perhaps due to inhibition of AKT signaling input, is responsible for lymphatic fate specification.

COUP-IIF has been shown to be required for PROX1 expression (31). Surprisingly, RAF1/ERK signaling induced PROX1 expression in arteries where COUP-TFII is not expressed, while suppression of the PI3K/AKT pathway inhibited both PROX1 and COUPTFII expression. This indicates that COUP-TFII might be required for PI3K/AKT dependency, but might not be essential for ERKinduced PROX1 expression.

During normal development, PROX1 induction is limited to the dorsolateral aspect of cardinal veins (17), demonstrating that it is a spatial context-dependent process. However, introduction of the mutant RAF1 $1^{\mathrm{S} 259 \mathrm{~A}}$ construct induced SOX18 and PROX1 expression throughout the entire circumference of the cardinal veins, obliterating the normal spatial gradient. This indicates 
that while RAF1/ERK signaling is able to regulate SOX18 and PROX1 expression, another factor(s) is responsible for the spatially polarized nature of this signal. Deactivation or suppression of PI3K/AKT signaling in the dorsolateral endothelium of cardinal veins may be one such mechanism.

While both SOX18 and PROX1 in S259A embryos were expressed throughout both venous and arterial endothelia, no significant induction of VEGFR3 or other LEC markers was observed in these vessels. This suggests that factors inhibiting lymphatic fate transition operate while ECs are part of blood vessels, and the full fate transition occurs only when they leave the vessel wall. This hypothesis is consistent with a recent study which showed that PROX1-positive LEC progenitors in cardinal veins do not express the lymphatic marker podoplanin prior to budding out of the vein (33).

The event responsible for the induction of ERK signaling in cardinal veins is not certain. Both VEGF-A and VEGF-C have the ability to activate PLC $\gamma /$ ERK pathway activity, respectively, via VEGFR2 and VEGFR3. Furthermore, both receptors are expressed in the cardinal vein at early embryonic stages. Previous studies have also shown that while VEGF-C is essential for the formation of lymphatic sprouts from embryonic veins, it is dispensable for cell commitment to the lymphatic endothelial lineage $(37,38)$. Moreover, mice with a VEGFR3 loss-of-ligandbinding mutation show defective lymphatic vessel growth, while jugular lymph sacs develop normally (19). Taken together, this suggests that neither VEGF-A nor VEGF-C is responsible for lymphatic fate commitment. It should also be noted that ERK activation in RAF1 $1^{\mathrm{S} 259 \mathrm{~A}}$ mutant embryos is due to the removal of baseline AKT inhibition of RAF1 and is not a direct activating effect of the transgene itself. Thus, the induction of Sox18 may depend as much on the withdrawal of AKT activation as on the direct activation of the RAF/ERK pathway.

Recent studies have shown that RAF1 functions in both a kinasedependent and -independent manner, with the latter not requiring ERK activation (39). The lymphatic phenotype in S259A embryos is due to RAF1-dependent activation of ERK, since expression of wild-type RAF1 in ECs has no significant effects on SOX18, PROX1, and other LEC markers. Furthermore, introduction of a constitutively active ERK construct (ME-LA) had a similar effect on LEC specification to RAF1 ${ }^{S 259 A}$. Finally, inhibition of ERK activation by an MEK inhibitor blocked RAF1 ${ }^{\mathrm{S} 259 \mathrm{~A}}$ induction of SOX18 expression. Overall, these results suggest that $R A F 1^{S 259 A}$ induces lymphatic specification in an ERK-dependent manner.

It is not clear how ERK regulates Sox18 levels. ERK is thought to regulate gene expression by phosphorylating various transcription factors; however, very little is known about transcriptional controls of Sox18 expression. A search for ERK-regulated transcription factor-binding sites in the Sox 18 promoter (no Sox18 enhancers have been described to date) identified, as expected, multiple possibilities including ETS1 and EGR1. While a recent study showed that EGR1 could activate the Sox18 promoter (40), it is not clear whether EGR1 is responsible for the effect described here. Further efforts will be required to fully understand Sox18 regulation by ERK signaling.

Gain-of-function mutations in the RAS signaling cascade, including those of RAF1, have been shown to cause Noonan syndrome and related disorders $(11,26)$. Common features of these "RASopathies" include increased ERK activation and lymphangiectasia $(41,42)$. Our data show that endothelial-specific expression of $R A F 1^{S 259 A}$ causes a lymphatic phenotype similar to that observed in Noonan and LEOPARD syndrome patients. Furthermore, inhibition of ERK activation in vivo reversed lymphangiectasia formation in S259A mice. Thus, endothelial ERK activation is responsible for the lymphatic phenotype observed in these patients, and suppression of ERK activation may be of therapeutic benefit.

In summary, we demonstrate the essential role of endothelial ERK signaling in lymphangiogenesis via induction of SOX18 and PROX1 expression in cardinal veins. Excessive ERK activation, induced by RAF1-activating mutations, induces excessive venous-to-lymphatic fate shift and is the molecular basis for lymphangiectasia and other lymphatic abnormalities seen in various "RASopathy" syndromes.

\section{Methods}

Cell culture. HUVECs and human microvascular EC-dLyAd-adult human dermal lymphatic microvascular ECs (HMVEC-dLyAd-HDLECs) were purchased from Lonza. HUVECs were cultured in M199 medium supplemented with $20 \% \mathrm{FBS}, 100 \mu \mathrm{g} / \mathrm{ml}$ ECGS, and $100 \mu \mathrm{g} / \mathrm{ml}$ heparin (Sigma-Aldrich). HDLECs were cultured in EBM-2MV medium (Lonza). A total of 293 cells were maintained in DMEM medium containing 10\% FBS.

Antibodies and reagents. The following antibodies were used for Western blotting: anti-pERK1/2, ERK1/2, pRAF1 S259, and RAF1 (Cell Signaling Technology); anti-VE-cadherin and CD31 (Santa Cruz Biotechnology); and anti-HA (Covance). Antibodies used for immunofluorescence staining were: anti-CD31 rabbit polyclonal; anti- $\beta$-gal (Abcam); anti-CD31 rat (BD Biosciences); anti-VEGFR3; anti-neuropilin 1; anti-COUP-TFII (R\&D Systems); anti-SMA (Sigma-Aldrich); anti-connexin 40 (Alpha Diagnostic International); anti-SOX18 (Aviva Systems Biology); antiPROX1 and anti-podoplanin (AngioBio); and anti-Ki67 (Dako). Secondary antibodies for immunoblotting were obtained from Sigma-Aldrich and from Invitrogen for immunofluorescence staining. Blocking reagent for immunofluorescence staining was from PerkinElmer. U0126 and rapamycin were obtained from Cell Signaling. LY294002 was from Sigma-Aldrich; AKT inhibitor VIII was from Calbiochem; and VEGF- $\mathrm{A}_{164}$ was obtained from R\&D Systems.

Transgenic mice. Human wild-type RAF1 and $R A F 1^{S 259 A}$ plasmids were gifts of Guri Tzivion (Wayne State University, Detroit, Michigan, USA). To generate $R A F 1^{S 259 A}$ transgenic mice, human $R A F 1^{5259 A}$ was cloned into the NotI-PstI sites of pBI-G Tet-off vector (Clontech). The TRE-RAF1 ${ }^{\text {S259A }}$ transgenic mouse line was generated at the Yale Animal Genomics Services Center. VE-cadherin-tTA mice (24) were from the W. Sessa Lab (Yale University). Timed matings were set up by crossing male TRE-RAF1 $1^{S 259 A}$ mice with $V E$-cadherin-tTA female mice. For paraffin and frozen sections, embryos were fixed in $4 \%$ paraformaldehyde overnight at $4{ }^{\circ} \mathrm{C}$.

$X$-gal staining. X-gal staining was performed using a $\beta$-Gal Expression kit from Millipore according to the manufacturer's instructions.

Immunohistochemistry. H\&E staining was performed as previously described (43). Immunofluorescence staining of paraffin sections $(7 \mu \mathrm{m})$ was performed by incubating primary and secondary antibodies in $0.5 \%$ blocking reagent in TNT buffer (50 mM Tris- $\mathrm{HCl} \mathrm{pH} 7.5,150 \mathrm{mM} \mathrm{NaCl}$, $0.05 \%$ Tween-20). Sections were then mounted using ProLong Gold antifade reagent from Invitrogen, and imaged.

Whole-mount immunofluorescence staining. Embryonic tissues were fixed in $4 \%$ PFA overnight at $4^{\circ} \mathrm{C}$, then washed 3 times with PBS. Tissues were permeabilized and blocked in TNBT buffer (100 mM Tris-HCl, $\mathrm{pH} 7.5$, $150 \mathrm{mM} \mathrm{NaCl}, 0.5 \%$ Triton-X100, and $0.05 \%$ blocking reagent) overnight at $4{ }^{\circ} \mathrm{C}$ and washed with TNT $(100 \mathrm{mM}$ Tris- $\mathrm{HCl}, \mathrm{pH} 7.5,150 \mathrm{mM} \mathrm{NaCl}$, $0.5 \%$ Triton-X100) buffer 6 times at room temperature. Tissues were then stained with primary antibodies at $4{ }^{\circ} \mathrm{C}$ overnight followed by fluorescent 
secondary antibodies in TNBT for 2 hours at room temperature. After washing 6 times with TNT buffer, tissues were mounted with ProLong Gold (Invitrogen) and imaged.

Image analysis. $\mathrm{H} \& \mathrm{E}$ images were acquired with a Nikon Eclipse 80i upright microscope. Fluorescence images were acquired using either a Leica SP5 confocal microscope or a PerkinElmer spinning disk confocal microscope. To image whole-mount immunofluorescence-stained samples, $z$-stack images with a $1-\mu \mathrm{m}$ step size were acquired on a Leica SP5 confocal microscope using LAS AF software. Three-dimensional projections were then assembled and vessel diameter analyzed using LAS AF software (Leica). Lumen area was quantified using ImageJ software (NIH).

Quantification of images. To quantify lumen areas of blood and lymphatic vessels, transverse paraffin sections of embryos at the lower neck position were stained with $\mathrm{H} \& \mathrm{E}$ or immunofluorescent antibodies, as indicated. Multiple images were then acquired using light microscopy. Lumen areas of vessels from 10 of every 6 consecutive $7-\mu \mathrm{m}$ sections of each embryo were then quantified using ImageJ software. The values were calculated as $\mu \mathrm{m}^{2}$ of the lumen area for each embryo, and the average for all embryos in the sample was then calculated. All data are presented as mean \pm SEM.

For skin vessel diameter quantification, lymphatic vessels in the dorsal skin of E14.5 embryos were visualized by VEGFR3 whole-mount immunofluorescence staining. Five independent 3D images of a $775 \mu \mathrm{m}$ $\times 775 \mu \mathrm{m}$ area of each skin sample per embryo were taken. Lymphatic vessel diameters were measured using LAS AF Lite imaging software (Leica).

To quantify PROX1-, SOX18-, and Ki67-positive cells, transverse paraffin sections of embryos at the lower neck position were stained with indicated antibodies. Positive cells from 10 of every 6 consecutive $7-\mu \mathrm{m}$ sections of each embryo were counted under the microscope. The numbers were then averaged to represent the mean number of each embryo per field.

Plasmids. HA-RAF1 WT and S259A mutant cDNA was PCR-amplified from PMT2-myc-RAF1 WT and S259A. PCR products were cloned into the NotI site of pLVX-IRES-Puro (Clontech) and pAd-Track-CMV to generate pLVX-HA-RAF1 WT, S259A and PAD-HA-RAF1 WT, S259A constructs. HA-RAF1 WT and S259A were cloned into pAdEasy-1 by recombination of PAD-HA-RAF1 WT, S259A, and pAdEasy-1 as previously described (44, 45). HA-ME and HA-ME-LA CDNAs were amplified by PCR from pEntry$M E$ and ME- $L A$, respectively. The PCR products were cloned into PENTER/ $\mathrm{D}-\mathrm{TOPO}$ (Invitrogen) and then recombined into the adenovirus vector $\mathrm{pAD} / \mathrm{CMV} / \mathrm{V} 5-\mathrm{DEST}$ as recommended (Invitrogen).

Production and infection of lentiviruses and adenoviruses. RAF1 lentiviruses were produced in $293 \mathrm{~T}$ cells (Invitrogen) by cotransfection of PLVX-HARAF1 WT or S259A with the lentiviral packaging plasmids pMD2.G, pRSVREV, and pMDLg/pRRE (Addgene) as described previously (44). Control lentiviruses were generated using the empty vector pLVX-IRES-puro. For lentiviral infection, cells were incubated with lentivirus in growth medium in the presence of $8 \mu \mathrm{g} / \mathrm{ml}$ polybrene (Sigma-Aldrich) for 8 hours. For adenoviral infection, cells were incubated with adenovirus $(\mathrm{MOI}=100)$ in growth medium overnight.

Quantitative RT-PCR and Western blotting. Total RNA was purified using an RNeasy Plus Mini kit (QIAGEN). cDNA was synthesized using the iScript cDNA Synthesis kit (Bio-Rad). Quantitative RT-PCR (qRT-PCR) was performed using iQ SYBR Green Supermix (Bio-Rad) on a Bio-Rad
CFX96 Real Time System. Primers used for qRT-PCR are listed in Supplemental Table 1. For Western blotting, cells were lysed in RIPA buffer supplemented with protease and phosphatase inhibitors. Cell lysates were then separated with $4 \%-20 \%$ gradient gel (Bio-Rad) and subjected to immunoblotting. Densitometry of Western blots was performed using ImageJ software (NIH).

Primary mouse EC isolation. Primary mouse ECs were isolated from mouse embryos or lungs using a protocol similar to that previously described (9). Briefly, embryos or lungs were harvested, minced finely with scissors, and then digested in $25 \mathrm{ml}$ collagenase $0.2 \%\left(\mathrm{w} / \mathrm{v}\right.$ ) at $37^{\circ} \mathrm{C}$ for 20 minutes (embryo) or 45 minutes (lung). The crude cell preparation was pelleted, resuspended in DPBS and then incubated with CD31-coated Dynabeads (Invitrogen) at room temperature for 10 minutes with rotation. Using a magnetic separator, the bead-bound cells were recovered and washed with DMEM 20\% FBS. The cells were then used for cell culture or gene expression analysis by qRT-PCR.

Administration of U0126 in mice. Timed matings were set up as described above. To investigate the effect of U0126 treatment on lymphatic vessel size, plugged mice were i.p. injected with either the MEK inhibitor U0126 $(5 \mathrm{mg} / \mathrm{kg}$ body weight) or the same volume of DMSO dissolved in PBS at 13.5 days post coitum. Twenty-four hours later, E14.5 embryos were harvested and lymphatic vasculature in the skin was visualized by VEGFR3 whole-mount staining. To investigate the effect of U0126 treatment on the expression of SOX18, PROX1, and other lymphatic markers, plugged mice were i.p. injected twice with either the MEK inhibitor U0126 $(5 \mathrm{mg} / \mathrm{kg}$ body weight) or the same volume of DMSO dissolved in PBS at E10.5 and E11.5 days post coitum, respectively. Forty-eight hours later, E12.5 embryos were harvested and subjected to further analysis.

Statistics. Differences between 2 groups were tested for statistical significance with a 2-tailed Student's $t$ test using SigmaPlot 11.0. A $P$ value less than 0.05 was considered significant.

Study approval. All the protocols and experiments performed in mice were approved by the Yale University School of Medicine.

\section{Acknowledgments}

We would like to thank Guri Tzivion (Wayne State University) for RAF1 constructs; William Sessa (Yale University) for VE-cadherintTA mice; Yan Huang (Yale University) for preparing adenoviruses; Nancy Ruddle (Yale University) and Mathias Francois (University of Queensland, Australia) for discussion; Rita Webber (Yale University) for taking care of mice; and Anthony Lanahan (Yale University) for help with manuscript editing. This study was supported in part by NIH grants HL84619 and HL107205 (to M. Simons), and a Leducq Foundation Transatlantic Network grant (to Y. Deng, A. Eichmann, and M. Simons).

Received for publication August 24, 2012, and accepted in revised form December 13, 2012.

Address correspondence to: Michael Simons, Section of Cardiovascular Medicine, Yale University School of Medicine, 333 Cedar St., New Haven, Connecticut 06510, USA. Phone: 203.785.7000; Fax: 203.785.5144; E-mail: michael.simons@yale.edu.
1. Alitalo K, Tammela T, Petrova TV. Lymphangiogenesis in development and human disease. Nature. 2005;438(7070):946-953.

2. Schulte-Merker S, Sabine A, Petrova TV. Lymphatic vascular morphogenesis in development, physiology, and disease. J Cell Biol. 2011;193(4):607-618.

3. Hosking B, Makinen T. Lymphatic vasculature: a molecular perspective. Bioessays. 2007; 29(12):1192-1202.
4. Wang Y, Oliver G. Current views on the function of the lymphatic vasculature in health and disease. Genes Dev. 2010;24(19):2115-2126.

5 . Faul JL, et al. Thoracic lymphangiomas, lymphangiectasis, lymphangiomatosis, and lymphatic dysplasia syndrome. Am J Respir Crit Care Med. 2000;161(3 Pt 1):1037-1046.

6. Adams RH, Alitalo K. Molecular regulation of angiogenesis and lymphangiogenesis. Nat Rev Mol
Cell Biol. 2007;8(6):464-478.

7. Aoki Y, Niihori T, Narumi Y, Kure S, Matsubara Y. The RAS/MAPK syndromes: novel roles of the RAS pathway in human genetic disorders. Hum Mutat. 2008;29(8):992-1006.

8. Tidyman WE, Rauen KA. The RASopathies: developmental syndromes of Ras/MAPK pathway dysregulation. Curr Opin Genet Dev. 2009;19(3):230-236.

9. Ren B, et al. ERK1/2-Akt1 crosstalk regulates 
arteriogenesis in mice and zebrafish. J Clin Invest. 2010;120(4):1217-1228.

10. Kuburovic V, Vukomanovic V, Carcavilla A, Ezquieta-Zubicaray B, Kuburovic N. Two cases of LEOPARD syndrome - RAF1 mutations firstly described in children. Turk J Pediatr. 2011;53(6):687-691.

11. Pandit B, et al. Gain-of-function RAF1 mutations cause Noonan and LEOPARD syndromes with hypertrophic cardiomyopathy. Nat Genet. 2007;39(8):1007-1012.

12. Ko JM, Kim JM, Kim GH, Yoo HW. PTPN11, SOS1, KRAS, and RAF1 gene analysis, and genotype-phenotype correlation in Korean patients with Noonan syndrome. J Hum Genet. 2008;53(11-12):999-1006.

13. Oliver G. Lymphatic vasculature development. Nat Rev Immunol. 2004;4(1):35-45

14. Srinivasan RS, et al. Lineage tracing demonstrates the venous origin of the mammalian lymphatic vasculature. Genes Dev. 2007;21(19):2422-2432.

15. Wigle JT, Oliver G. Prox 1 function is required for the development of the murine lymphatic system. Cell. 1999;98(6):769-778.

16. Francois M, Harvey NL, Hogan BM. The transcriptional control of lymphatic vascular development. Physiology (Bethesda). 2011;26(3):146-155.

17. Francois M, et al. Sox 18 induces development of the lymphatic vasculature in mice. Nature. 2008;456(7222):643-647.

18. Hosking B, et al. Sox7 and Sox17 are strain-specific modifiers of the lymphangiogenic defects caused by Sox 18 dysfunction in mice. Development. 2009;136(14):2385-2391.

19. Zhang L, et al. VEGFR-3 ligand-binding and kinase activity are required for lymphangiogenesis but not for angiogenesis. Cell Res. 2010;20(12):1319-1331.

20. Makinen T, et al. Isolated lymphatic endothelial cells transduce growth, survival and migratory signals via the VEGF-C/D receptor VEGFR-3. EMBOJ. 2001;20(17):4762-4773.

21. Zhou F, et al. Akt/protein kinase B is required for lymphatic network formation, remodeling, and valve development. Am J Pathol. 2010;177(4):2124-2133.

22. Taniguchi K, et al. Spreds are essential for embry- onic lymphangiogenesis by regulating vascular endothelial growth factor receptor 3 signaling. $\mathrm{Mol}$ Cell Biol. 2007;27(12):4541-4550.

23. Lapinski PE, et al. RASA1 maintains the lymphatic vasculature in a quiescent functional state in mice. J Clin Invest. 2012;122(2):733-747.

24. Sun JF, et al. Microvascular patterning is controlled by fine-tuning the Akt signal. Proc Natl Acad SciU S A. 2005;102(1):128-133.

25. von Gise A, et al. YAP1, the nuclear target of Hippo signaling, stimulates heart growth through cardiomyocyte proliferation but not hypertrophy. Proc Natl Acad Sci U S A. 2012;109(7):2394-2399.

26. Razzaque MA, et al. Germline gain-of-function mutations in RAF1 cause Noonan syndrome. Nat Genet. 2007;39(8):1013-1017.

27. Petrova TV, et al. Lymphatic endothelial reprogramming of vascular endothelial cells by the Prox-1 homeobox transcription factor. EMBO J. 2002;21(17):4593-4599.

28. Johnson NC, et al. Lymphatic endothelial cell identity is reversible and its maintenance requires Prox 1 activity. Genes Dev. 2008;22(23):3282-3291.

29. Oliver G, Srinivasan RS. Endothelial cell plasticity: how to become and remain a lymphatic endothelial cell. Development. 2010;137(3):363-372.

30. Hong YK, et al. Prox1 is a master control gene in the program specifying lymphatic endothelial cell fate. Dev Dyn. 2002;225(3):351-357.

31. Srinivasan RS, et al. The nuclear hormone receptor Coup-TFII is required for the initiation and early maintenance of Prox 1 expression in lymphatic endothelial cells. Genes Dev. 2010;24(7):696-707.

32. Srinivasan RS, Oliver G. Prox1 dosage controls the number of lymphatic endothelial cell progenitors and the formation of the lymphovenous valves. Genes Dev. 2011;25(20):2187-2197.

33. Yang $Y$, et al. Lymphatic endothelial progenitors bud from the cardinal vein and intersomitic vessels in mammalian embryos. Blood. 2012;120(11):2340-2348.

34. Wu X, et al. MEK-ERK pathway modulation ameliorates disease phenotypes in a mouse model of Noonan syndrome associated with the Raf1(L613V) mutation. J Clin Invest. 2011; 121(3):1009-1025.

35. Allanson JE, Roberts AE. Noonan syndrome. In: Pagon RA, Bird TD, Dolan CR, Stephens K, Adam MP, eds. Gene Reviews. Seattle, Washington, USA: University of Washington; 2001.

36. Mouta-Bellum C, et al. Organ-specific lymphangiectasia, arrested lymphatic sprouting, and maturation defects resulting from gene-targeting of the PI3K regulatory isoforms p85alpha, p55alpha, and p50alpha. Dev Dyn. 2009;238(10):2670-2679.

37. Karkkainen MJ, et al. Vascular endothelial growth factor $\mathrm{C}$ is required for sprouting of the first lymphatic vessels from embryonic veins. Nat Immunol. 2004;5(1):74-80.

38. Haiko P, et al. Deletion of vascular endothelial growth factor C (VEGF-C) and VEGF-D is not equivalent to VEGF receptor 3 deletion in mouse embryos. Mol Cell Biol. 2008;28(15):4843-4850.

39. Mielgo A, et al. A MEK-independent role for CRAF in mitosis and tumor progression. Nat Med. 2011;17(12):1641-1645.

40. Petrovic I, Kovacevic-Grujicic N, Stevanovic M. Early growth response protein 1 acts as an activator of SOX18 promoter. Exp Mol Med. 2010; 42(2):132-142.

41. Ozturk S, et al. A case of Noonan syndrome with pulmonary and abdominal lymphangiectasia. Int $J$ Clin Pract. 2000;54(4):274-276.

42. Herzog DB, Logan R, Kooistra JB. The Noonan syndrome with intestinal lymphangiectasia. J Pediatr. 1976;88(2):270-272.

43. Kiernan JA. Histological and Histochemical Methods: Theory and Practice. 4th ed. Cold Spring Harbor, New York, USA: Cold Spring Harbor Laboratory Press; 2008.

44. Dull T, et al. A third-generation lentivirus vector with a conditional packaging system. J Virol. 1998;72(11):8463-8471.

45. Luo J, et al. A protocol for rapid generation of recombinant adenoviruses using the AdEasy system. Nat Protoc. 2007;2(5):1236-1247. 